\title{
G-twisted braces and orbifold Landau-Ginzburg Models
}

\author{
Weiqiang $\mathrm{He}, \mathrm{Si} \mathrm{Li}$ and Yifan Li
}

\begin{abstract}
Given an algebra with group $G$-action, we construct brace structures for its $G$-twisted Hochschild cochains. An an application, we construct $G$-Frobenius algebras for orbifold Landau-Ginzburg Bmodels and present explicit orbifold cup product formula for all invertible polynomials.
\end{abstract}

\section{Contents}

1 Introduction

$2 G$-twisted Hochschild complexes for curved algebras 4

2.1 Hochschild cochains and brace structure . . . . . . . . . . . 5

2.2 Curved algebras and mixed complex $\ldots \ldots \ldots \ldots \ldots \ldots \ldots$

$2.3 G$-curved algebras and $G$-twisted brace structures $\ldots \ldots \ldots \ldots \ldots$

2.4 A comparison between Gerstenhaber algebras . . . . . . . . . . . . 12

3 Orbifold Landau-Ginzburg B-models 14

3.1 Bar resolution vs. Koszul resolution . . . . . . . . . . . . . . . . . . . 14

$3.2 G$-twisted cohomology . . . . . . . . . . . . . . . . 20

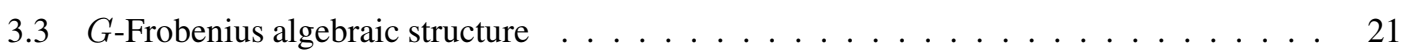

3.4 Quantum differential operator and cup product . . . . . . . . . . . . . . . . 24

3.5 Invertible polynomials and cup product formula . . . . . . . . . . . . . 29

\section{Introduction}

Consider a triple $(A, W, G)$ (we call $G$-twisted curved algebra), where $A$ is an associative algebra with a compatible $G$-action and $W$ is a $G$-invariant central element of $A$. Associated to $A$, we have Hochschild cochains $C^{\bullet}(A, A)$ where many homological constructions are captured by brace structures [19, 20, 52]. In this paper, we generalize such brace structures to $G$-twisted Hochschild cochains $C^{\bullet}(A, A[G])$ (Definition 2.10) and prove a $G$-twisted version of higher pre-Jacobi identities (Proposition 2.2).

Let

$$
\partial_{H}: C^{\bullet}(A, A[G]) \rightarrow C^{\bullet+1}(A, A[G])
$$


be the Hochschild differential. Let $\mathrm{HH}^{\bullet}(A, A[G])$ be the Hochschild cohomology with a natural cup product $\cup$. We show that $\cup$ satisfies a $G$-twisted commutativity relation (see also $[46]$ ) and the $G$-invariant subspace $\mathrm{HH}^{\bullet}(A, A[G])^{G}$ is a Gerstenhaber algebra (Theorem 2.7). When $G$ is a finite group, a result of Ştefan (Cor. 3.4 in [48]) implies that $\mathrm{HH}^{\bullet}(A[G], A[G])$ and $\mathrm{HH}^{\bullet}(A, A[G])^{G}$ are isomorphic as graded vector spaces (see also [11]). Using $G$-twisted brace structures, we extend this to an isomorphism as Gerstenhaber algebras (Theorem 2.10).

Let $W$ be a $G$-invariant central element of $A$. It leads to a curving differential (Definition 2.7)

$$
\mathrm{d}_{W}: C^{\bullet}(A, A[G]) \rightarrow C^{\bullet-1}(A, A[G])
$$

such that $\left(C^{\bullet}(A, A[G]), \partial_{H}, \mathrm{~d}_{W}\right)$ forms a mixed complex. All the above constructions apply to $\left(\partial_{H}+\right.$ $\left.\mathrm{d}_{W}\right)$-cohomologies which are instead $\mathbb{Z} / 2 \mathbb{Z}$-graded.

Our study of $(A, G, W)$ is motivated by mirror symmetry between two singularity theories: LandauGinzburg (LG) A-model and Landau-Ginzburg (LG) B-model. Such LG/LG mirror symmetry is parallel to the well-studied Calabi-Yau/Calabi-Yau and Toric/Landau-Ginzburg mirror symmetry. The basic data for LG model consists of $(A, W, G)$ where $W$ is a holomorphic function (called the superpotential)

$$
W: X \rightarrow \mathbb{C}
$$

on a complex variety $X$ and $G$ is a group (called the orbifold group) acting on $X$ preserving $W . A=$ $\mathcal{O}(X)$ is the structure ring of $X$. A version of compact type Hochschild cohomology ([25, 36, 38], see Definition 2.13) turns out to be relevant for the state spaces of orbifold LG models [51, 26].

In this paper we focus on the case when $W: \mathbb{C}^{N} \rightarrow \mathbb{C}$ is a weighted homogeneous polynomial

$$
W\left(\lambda^{q_{i}} x_{i}\right)=\lambda W\left(x_{i}\right), \quad \forall \lambda \in \mathbb{C}^{*}
$$

with an isolated critical point at the origin and contains no monomials of the form $x^{i} x^{j}$ for $i \neq j$. Here $q_{i} \in\left(0, \frac{1}{2}\right] \cap \mathbb{Q}$ is called the weight of $x_{i}$. The orbifold group $G$ will be a subgroup of $G_{W}$ where

$$
G_{W}=\left\{\left(\lambda_{1}, \ldots, \lambda_{N}\right) \in\left(\mathbb{C}^{\times}\right)^{N} \mid W\left(\lambda_{1} x_{1}, \ldots, \lambda_{N} x_{N}\right)=W\left(x_{1}, \ldots, x_{N}\right)\right\} .
$$

There exists a construction of LG/LG mirror pairs which originates from a physical construction of Berglund-Hübsch [4] and is further completed by Krawitz [31]. In this construction, the polynomial $W$ is required to be invertible [33, 8, 9], i.e., the number of variables must equal to the number of monomials of $W$. By rescaling the variables, we can always write $W$ as

$$
W=\sum_{i=1}^{N} \prod_{j=1}^{N} x_{j}^{a_{i j}} .
$$

We denote its exponent matrix by $E_{W}=\left(a_{i j}\right)_{N \times N}$. The mirror polynomial of $W$ is

$$
W^{T}=\sum_{i=1}^{N} \prod_{j=1}^{N} x_{j}^{a_{j i}},
$$

i.e., the exponent matrix $E_{W^{T}}$ of the mirror polynomial is the transpose matrix of $E_{W}$. The mirror construction between orbifold groups $G^{T} \subset G_{W^{T}}$ and $G \subset G_{W}$ is more involved but explicitly known. It has the property that bigger $G$ corresponds to smaller $G^{T}$ and vice versa.

A general mirror theorem is proved (see[24] and references therein for related works) between LG Amodel (FJRW theory [15]) of $\left(W^{T}, G_{W^{T}}\right)$ and LG B-model (Saito-Givental theory [39, 21]) of ( $W, G=$ trivial). Correlation functions for LG B-model when $G$ is not a trivial group is less known. 
As an application of methods developed in this paper, we construct $G$-Frobenius algebraic structure in the sense of Kaufmann [29, 30] for orbifold Landau-Ginzburg B-models (Theorem 3.11] (see also [3] for certain axiomatic discussion of Kaufman's definition in orbifold LG models). Moreover, it is the first time we are able to compute explicit orbifold cup product formula for all invertible polynomials. This computation is based on a construction of explicit homotopy retract (Section 3.1)

$$
\tilde{\mathrm{H}}^{*} \bigodot\left(C^{\bullet}(\bar{A}, A[G]), \partial_{H}+\mathrm{d}_{W}\right) \underset{\Upsilon^{*}}{\stackrel{\tilde{\Phi}^{*}}{\longleftarrow}}\left(K^{\bullet}(A, A[G]), \partial_{K}+\tilde{\mathrm{d}}_{W}\right)
$$

between $G$-twisted reduced Hochschild complex $C^{\bullet}(\bar{A}, A[G])$ and $G$-twisted Koszul complex $K^{\bullet}(A, A[G])$. This homotopy retract is obtained from a version of homological perturbation from a construction of Shepler and Witherspoon [42] in the case without $W$. The result is summarized as follows.

According to [32], an invertible polynomial is classified as a direct sum of three types of elementary invertible polynomials:

(a) Fermat type: $W=x_{1}^{n}$.

(b) Loop type: $W=x_{1}^{n_{1}} x_{2}+x_{2}^{n_{2}} x_{3}+\cdots+x_{N}^{n_{N}} x_{1}$.

(c) Chain type: $W=x_{1}^{n_{1}} x_{2}+x_{2}^{n_{2}} x_{3}+\cdots+x_{N}^{n_{N}}$.

Let $g \in G_{W}, g\left(x_{i}\right)=\lambda_{i} x_{i}$ where not all $\lambda_{i}$ 's are 1 . We define a version of $g$-twisted Hessian Hess $^{g}(W)$ (Definition (3.10) ). For example, for loop type polynomial, the $g$-twisted Hessian has the form

$$
\operatorname{Hess}^{g}(W)=\frac{(-1)^{N+1}+n_{1} n_{2} \cdots n_{N}}{\left(1-\lambda_{1}\right)\left(1-\lambda_{2}\right) \cdots\left(1-\lambda_{N}\right)} x_{1}^{n_{1}-1} x_{2}^{n_{2}-1} \cdots x_{N}^{n_{N}-1} .
$$

Let $1_{g}$ be the generator of the $g$-twisted sector (see (3.33). Then our orbifold cup product formula reads

$$
\mathbf{1}_{g} \cup \mathbf{1}_{g^{-1}}=(-1)^{\frac{N(N-1)}{2}} \operatorname{Hess}^{g}(W) \mathbf{1}_{\mathrm{e}}
$$

where e $\in G$ is the identity. This explicitly determines the full orbifold cup product (Theorem 3.20).

Alternatively, there exists categorical approach to orbifold Landau-Ginzburg models in terms of the dg-category $\operatorname{MF}(A[G], W)$ of matrix factorizations. In the case when $G$ is trivial, Hochschild cohomology is computed by Dyckerhoff in terms of compact generators [12]. Alternately, there is another approach via curved algebra which we follow in this paper. In [36], Polishchuk and Positselski identify Hochschild cohomology and compact type Hochschild cohomology of $\operatorname{MF}(A, W)$. Segal [40] constructs a quasi-isomorphism between compact type Hochschild complex of $\operatorname{MF}(A, W)$ and that of the curved algebra $(A, W)$. Căldăraru and Tu compute the compact type Hochschild (co)-homology of $(A, W)$ explicitly in [6]. In the orbifold case when $G$ is nontrivial, Hochschild cohomology is computed in terms of compact generators [37] or directly in terms of curved algebras [6, 40]. These two approaches are identified in [50]. Based on the result of [36], Shklyarov [46] shows that the categorical cup product of matrix factorizations is identical to the Hochschild cup product of $G$-twisted curved algebras.

On the other hand, it is very difficult to compute orbifold cup product through categories. In [46], Shklyarov deduces a formula of cup product in terms of certain complicated unknown coefficient $\sigma_{g, h}$. In appendix of [46], Basalaev and Shklyarov actually obtain interesting closed formula of cup product in some special cases (two variable chain polynomial and Fukaya category of surface).

In contrast to Shklyarov's approach, we use an explicit homotopy retract between Hochschild complex and Koszul complex to deduce a combinatorial formula. This allows us to explicitly compute orbifold 
cup product for all invertible polynomials. Our result confirms a conjecture by Basalaev and Shklyarov in Appendix A of [46]. It is a very interesting question to compare our results with Shklyarov's formula.

Acknowledgements. We would like to thank Xiaojun Chen, Yu Liu, Junwu Tu, Bin Wang, Guodong Zhou for helpful discussions. S. L. is partially supported by Grant 20151080445 of Independent Research Program at Tsinghua University. W. H. is partially supported by Tsinghua Postdoc Grant 100410019.

\section{II $G$-twisted Hochschild complexes for curved algebras}

In this paper we fix a field $k$ of characteristic zero. All vector spaces and algebras are defined over $k$. Let $V$ be a $\mathbb{Z}$ or $\mathbb{Z} / 2 \mathbb{Z}$-graded $k$-vector space. Let $V_{d}=\{x \in V|| x \mid=d\} \subset V$ denote the subspace of degree $d$ elements. Let $V[m]$ denote the degree shifting by $m$ such that

$$
V[m]_{d}=V_{d+m} .
$$

When $V$ is $\mathbb{Z} / 2 \mathbb{Z}$-graded, the above degree shifting is understood modulo $2 \mathbb{Z}$. For example

$$
V[1]_{\overline{0}}=V_{\overline{1}}, \quad V[1]_{\overline{1}}=V_{\overline{0}}, \quad \text { where } \quad \overline{0}, \overline{1} \in \mathbb{Z} / 2 \mathbb{Z} .
$$

Definition 2.1. A curved algebra is a pair $(A, W)$ where $A$ is an associative algebra and $W$ is a central element of $A$. We will sometimes denote the pair $(A, W)$ for a curved algebra by $A_{W}$.

Definition 2.2. Let $G$ be a group. A $G$-twisted curved algebra is a triple $(A, W, G)$ where $A$ is an associative algebra with $G$-equivariant product and $W$ is a $G$-invariant central element of $A . \forall g \in G$ and $a \in A$, we denote by ${ }^{g} a$ the left action of $a$ by $g$. The identity element of $G$ is always denoted by e.

Definition 2.3. Given a $G$-twisted curved algebra $(A, W, G)$, we define its $G$-orbifolding to be the $G$ twisted curved algebra $(A[G], W, G)$ where

(a) $A[G]=A \otimes_{k} k[G]$ is the crossed product algebra where $k[G]$ is the group algebra. The product is

$$
\left(a_{1} g_{1}\right) \cdot\left(a_{2} g_{2}\right):=\left(a_{1} \cdot{ }^{g_{1}} a_{2}\right) g_{1} g_{2}, \quad \text { where } \quad a_{1}, a_{2} \in A, g_{1}, g_{2} \in G .
$$

(b) $G$-action:

$$
\begin{aligned}
G \times A[G] & \rightarrow A[G] \\
\left(g, a g^{\prime}\right) & \mapsto g \cdot\left(a g^{\prime}\right):=\left({ }^{g} a\right) g g^{\prime} g^{-1}
\end{aligned}
$$

(c) $G$-invariant central element $=W$ e.

In this section, we construct $G$-twisted brace structures (Definition 2.10) on $G$-twisted Hochschild cochains (Definition 2.9). This generalizes the usual brace operations on Hochschild cochains of associative algebras. As an application, we obtain a $G$-twisted commutative structure on $G$-twisted Hochschild cohomology (Theorem 2.7) and a comparison theorem between two versions of $G$-twisted Gerstenhaber algebras (Theorem 2.10 and Theorem 2.11). This will be applied in the next section to establish our main results on $G$-Frobenius algebras of orbifold Landau-Ginzburg models. 


\subsection{Hochschild cochains and brace structure}

Definition 2.4. Given an associative algebra A, we denote Hochschild cochains and compact type Hochschild cochains of $A$ by

$$
C^{\bullet}(A, A)=\prod_{p=0}^{\infty} C^{p}(A, A), \quad C_{c}^{\bullet}(A, A)=\bigoplus_{p=0}^{\infty} C^{p}(A, A)
$$

where

$$
C^{p}(A, A)=\operatorname{Hom}_{k}\left(A^{\otimes p}, A\right) .
$$

Given $\phi \in C^{p}(A, A)$, we write $|\phi|=p$ for its degree.

Following [19, 20, 17, 7, 49], we can define higher operations on Hochschild cochains as a generalization of Gerstenhaber product introduced in [16]. Given $\phi \in C^{p}(A, A), \phi_{i} \in C^{p_{i}}(A, A)$ for $i=1, \cdots, k$ and $a_{1}, a_{2} \cdots \in A$, we define (if $k \leq p$ )

$$
\begin{aligned}
& \phi\left\{\phi_{1}, \phi_{2}, \cdots \phi_{k}\right\}\left(a_{1} \otimes a_{2} \otimes \cdots\right) \\
&=\sum_{\boldsymbol{I} \in \mathcal{I}}(-1)^{\sum_{j=1}^{k}\left(i_{j}-1\right)\left(\left|\phi_{j}\right|-1\right)} \phi\left(a_{1} \otimes \cdots \otimes a_{i_{1}-1} \otimes \phi_{1}\left(a_{i_{1}} \otimes \cdots\right) \otimes \cdots\right. \\
&\left.\cdots \otimes a_{i_{j}-1} \otimes \phi_{j}\left(a_{i_{j}} \otimes \cdots\right) \otimes \cdots\right)
\end{aligned}
$$

where

$$
\mathcal{I}=\left\{\boldsymbol{I}=\left(i_{1}<i_{2}<\cdots<i_{k}\right) \mid i_{1}>0 \text { and } \forall 1 \leqslant j<k, i_{j}+\left|\phi_{j}\right| \leqslant i_{j+1}\right\} .
$$

If $k>p$, we set $\phi\left\{\phi_{1}, \phi_{2}, \cdots \phi_{k}\right\}=0$. The map

$$
\begin{aligned}
C^{\bullet}(A, A) & \rightarrow C^{\bullet}\left(C^{\bullet}(A, A), C^{\bullet}(A, A)\right) \\
\phi & \mapsto \phi\{\cdots\}
\end{aligned}
$$

gives brace structures [19, 20, 52]. It satisfies the following higher pre-Jacobi identity

$$
\begin{aligned}
& \phi\left\{\phi_{1}, \phi_{2} \cdots \phi_{n}\right\}\left\{\psi_{1}, \psi_{2} \cdots \psi_{m}\right\} \\
= & \sum \pm \phi\left\{\psi_{1} \cdots \psi_{j_{1}-1}, \phi_{1}\left\{\psi_{j_{1}} \cdots\right\} \cdots \psi_{j_{n}-1}, \phi_{n}\left\{\psi_{j_{n}} \cdots\right\} \cdots \psi_{m}\right\} .
\end{aligned}
$$

When $k=1$, we get the Gerstenhaber product

$$
\phi\left\{\phi_{1}\right\}=\phi \circ \phi_{1} .
$$

Pre-Jacobi identity implies that the Gerstenhaber bracket on $C^{\bullet}(A, A)$ defined by

$$
\left\{\phi_{1}, \phi_{2}\right\}=\phi_{1}\left\{\phi_{2}\right\}-(-1)^{\left(\left|\phi_{1}\right|-1\right)\left(\left|\phi_{2}\right|-1\right)} \phi_{2}\left\{\phi_{1}\right\}, \quad \forall \phi_{1}, \phi_{2} \in C^{\bullet}(A, A),
$$

gives a graded Lie algebra structure on $C^{\bullet}(A, A)[1]$.

The product · on $A$ gives rise to a Hochschild cochain

$$
m_{2} \in C^{2}(A, A), \quad m_{2}\left(a_{1}, a_{2}\right)=a_{1} \cdot a_{2}, \quad a_{i} \in A .
$$

The associativity of the product is equivalent to

$$
\left\{m_{2}, m_{2}\right\}=0 .
$$


Definition 2.5. The cup product $\cup$ and Hochschild differential $\partial_{H}$ on $C^{\bullet}(A, A)$ are defined by

$$
\phi_{1} \cup \phi_{2}=(-1)^{\left|\phi_{1}\right|\left(\left|\phi_{2}\right|-1\right)} m_{2}\left\{\phi_{1}, \phi_{2}\right\}, \quad \partial_{H}(\phi):=(-1)^{|\phi|-1}\left\{m_{2}, \phi\right\} .
$$

Higher pre-Jacobi identity for brace operations implies the following higher homotopies,

$$
\begin{aligned}
& \partial_{H}(\phi)\left\{\phi_{1}, \phi_{2}, \cdots, \phi_{n}\right\} \\
= & (-1)^{\xi_{n}} \partial_{H}\left(\phi\left\{\phi_{1}, \cdots, \phi_{n}\right\}\right)-\sum_{k=1}^{n}(-1)^{\xi_{k}} \phi\left\{\phi_{1}, \cdots, \phi_{k-1}, \partial_{H}\left(\phi_{k}\right), \phi_{k+1}, \cdots, \phi_{n}\right\} \\
& +(-1)^{\left|\phi_{1}\right| \xi_{1}^{\prime}} \phi_{1} \cup \phi\left\{\phi_{2}, \phi_{3}, \cdots, \phi_{n}\right\}-(-1)^{|\phi|\left|\phi_{n}\right|+\xi_{n-1} \xi_{n-1}^{\prime} \phi\left\{\phi_{1}, \phi_{2}, \cdots, \phi_{n-1}\right\} \cup \phi_{n}} \\
& +\sum_{k=1}^{n-1}(-1)^{\xi_{k}+\left|\phi_{k}\right|\left|\phi_{k+1}\right|} \phi\left\{\phi_{1}, \cdots, \phi_{k} \cup \phi_{k+1}, \cdots, \phi_{n}\right\},
\end{aligned}
$$

with

$$
\left\{\begin{array}{l}
\xi_{k}=\left|\phi_{1}\right|+\left|\phi_{2}\right|+\cdots+\left|\phi_{k}\right|-k, \\
\xi_{k}^{\prime}=\left|\phi_{k+1}\right|+\left|\phi_{k+2}\right|+\cdots+\left|\phi_{n}\right|-(n-k) .
\end{array}\right.
$$

Here are some identities for lower braces

- $n=1$. We find

$$
(-1)^{\left|\phi_{1}\right|} \partial_{H}\left(\phi\left\{\phi_{1}\right\}\right)+\left(\partial_{H} \phi\right)\left\{\phi_{1}\right\}-(-1)^{\left|\phi_{1}\right|} \phi\left\{\partial_{H} \phi_{1}\right\}=\phi_{1} \cup \phi-(-1)^{|\phi|\left|\phi_{1}\right|} \phi \cup \phi_{1} \text {. }
$$

This says that $\cup$ on $C^{\bullet}(A, A)$ is commutative up to homotopy. Switching the role of $\phi, \phi_{1}$ and comparing the difference, we find the compatibility of $\partial_{H}$ with the Gerstenhaber bracket [16],

$$
\partial_{H}\left\{\phi, \phi_{1}\right\}=(-1)^{\left|\phi_{1}\right|-1}\left\{\partial_{H} \phi, \phi_{1}\right\}+\left\{\phi, \partial_{H} \phi_{1}\right\} .
$$

- $n=2$. We find

$$
\begin{aligned}
& \partial_{H}\left(\phi\left\{\phi_{1}, \phi_{2}\right\}\right)-(-1)^{\left|\phi_{2}\right|-1} \phi\left\{\partial_{H} \phi_{1}, \phi_{2}\right\}-\phi\left\{\phi_{1}, \partial_{H} \phi_{2}\right\}-(-1)^{\left|\phi_{1}\right|+\left|\phi_{2}\right|} \partial_{H}(\phi)\left\{\phi_{1}, \phi_{2}\right\} \\
= & (-1)^{\left(\left|\phi_{1}\right|-1\right)\left|\phi_{2}\right|}\left(\phi\left\{\phi_{1} \cup \phi_{2}\right\}-(-1)^{(|\phi|-1)\left|\phi_{2}\right|} \phi\left\{\phi_{1}\right\} \cup \phi_{2}-\phi_{1} \cup \phi\left\{\phi_{2}\right\}\right) .
\end{aligned}
$$

Set $\phi=m_{2}$ and use $m_{2}\left\{m_{2}\right\}=0$, we find

$$
\partial_{H}\left(\phi_{1} \cup \phi_{2}\right)=\partial_{H}\left(\phi_{1}\right) \cup \phi_{2}+(-1)^{\left|\phi_{1}\right|} \phi_{1} \cup \partial_{H}\left(\phi_{2}\right) \text {. }
$$

This says that the triple

$$
\left(C^{\bullet}(A, A), \partial_{H}, \cup\right)
$$

defines a differential graded algebra (dga).

Definition 2.6. A Gerstenhaber algebra $(A, \cdot,\{\}$,$) is a \mathbb{Z}$ (or $\mathbb{Z} / 2 \mathbb{Z})$-graded commutative algebra with a graded Lie structure on $A[1]$ satisfying the shifted Poisson identities. Thus, for $a, b, c \in A$, we have

- $|a \cdot b|=|a|+|b|$ and $a \cdot b=(-1)^{|a||b|} b \cdot a$.

- $|\{a, b\}|=|a|+|b|-1,\{a, b\}=-(-1)^{(|a|-1)(|b|-1)}\{b, a\}$ and $\{a,\{b, c\}\}=\{\{a, b\}, c\}+$ $(-1)^{(|a|-1)(|b|-1)}\{b,\{a, c\}\}$.

- $\{a \cdot b, c\}=\{a, b\} \cdot c+(-1)^{|a|(|c|-1)} a \cdot\{b, c\}$.

The direct consequence of (2.5) (2.8) 2.9) 2.10) says that the Hochschild cohomology

$$
\mathrm{HH}^{\bullet}(A):=\mathrm{H}^{\bullet}\left(C^{\bullet}(A, A), \partial_{H}\right)
$$

together with the cup product and Gerstenhaber bracket form a Gerstenhaber algebra. 


\subsection{Curved algebras and mixed complex}

We consider a curved algebra $(A, W)$ where $W$ is a central element of $A$. The curving $W$ defines

$$
m_{0} \in C^{0}(A, A), \quad m_{0}(1)=W .
$$

Definition 2.7. We define the curving differential $\mathrm{d}_{W}$ on Hochschild cochains of $A$ by

$$
\mathrm{d}_{W}=(-1)^{p}\left\{m_{0},-\right\}: C^{p}(A, A) \rightarrow C^{p-1}(A, A)
$$

and the curved Hochschild differential by

$$
\partial_{H}^{W}=\partial_{H}+\mathbf{d}_{W}
$$

The following identities hold

$$
\partial_{H}^{2}=\mathrm{d}_{W}^{2}=\left(\partial_{H}^{W}\right)^{2}=0 .
$$

Therefore the triple $\left\{C^{\bullet}(A, A), \partial_{H}, \mathrm{~d}_{W}\right\}$ defines a mixed complex. We are interested in the cohomology for the mixed differential $\partial_{H}^{W}$, which is sensitive to the topology we use. Following [5, 6, 40], the appropriate complex for Landau-Ginzburg models turns out to be the one of compact type above as induced in [25, 36, 38].

Definition 2.8. We define the compact type Hochschild cohomology for a curved algebra $(A, W)$ by

$$
\mathrm{HH}_{c}\left(A_{W}\right)=\mathrm{H}\left(C_{c}^{\bullet}(A, A), \partial_{H}^{W}\right) .
$$

$\mathrm{HH}_{c}\left(A_{W}\right)$ is $\mathbb{Z} / 2 \mathbb{Z}$-graded in terms of the parity of the degree

$$
\mathrm{HH}_{c}\left(A_{W}\right)=\mathrm{HH}_{c}^{\overline{0}}\left(A_{W}\right) \oplus \mathrm{HH}_{c}^{\overline{1}}\left(A_{W}\right) .
$$

Proposition 2.1. $\mathrm{HH}_{c}\left(A_{W}\right)$ is a $\mathbb{Z} / 2 \mathbb{Z}$-graded Gerstenhaber algebra.

Proof. Equations (2.8) 2.9) 2.10) 2.11) still hold if $\partial_{H}$ is replaced by $\partial_{H}^{W}$. It follows that the cup product $\cup$ and the Gerstenhaber bracket $\{-,-\}$ induce the Gerstenhaber algebra structure on $\mathrm{HH}_{c}\left(A_{W}\right)$.

\section{3 $G$-curved algebras and $G$-twisted brace structures}

Definition 2.9. Let $(A, W, G)$ be a $G$-twisted curved algebra. We define the G-twisted Hochschild cochains and compact type Hochschild cochains by

$$
C^{\bullet}(A, A[G])=\prod_{p=0}^{\infty} C^{p}(A, A[G]), \quad C_{c}^{\bullet}(A, A[G])=\bigoplus_{p=0}^{\infty} C^{p}(A, A[G])
$$

where

$$
C^{p}(A, A[G])=\operatorname{Hom}_{k}\left(A^{\otimes p}, A[G]\right)
$$

There is a natural $G$-action on $G$-twisted Hochschild cochains

$$
\begin{aligned}
G \times C^{p}(A, A[G]) & \rightarrow C^{p}(A, A[G]), \\
(g, \phi) & \mapsto g^{*}(\phi),
\end{aligned}
$$

given by

$$
g^{*}(\phi)\left(a_{1} \otimes a_{2} \otimes \cdots \otimes a_{p}\right)=g \cdot \phi\left(^{g^{-1}} a_{1} \otimes^{g^{-1}} a_{2} \otimes \cdots \otimes g^{-1} a_{p}\right) \cdot g^{-1} .
$$




\section{$G$-twisted braces}

Definition 2.10 ( $G$-twisted braces). Given $\phi \in C^{p}(A, A g), \phi_{i} \in C^{p_{i}}\left(A, A g_{i}\right)$ for $i=1, \cdots, k$ and $a_{1}, a_{2} \cdots \in A$, we define (if $k \leq p$ )

$$
\begin{gathered}
\phi\left\{\phi_{1}, \phi_{2}, \cdots \phi_{k}\right\}\left(a_{1} \otimes a_{2} \otimes \cdots\right) \\
:=\sum_{\boldsymbol{I} \in \mathcal{I}}(-1)^{\sum_{j=1}^{k}\left(i_{j}-1\right)\left(\left|\phi_{j}\right|-1\right)} \phi^{\circ}\left(a_{1} \otimes \cdots \otimes a_{i_{1}-1} \otimes \phi_{1}^{\circ}\left(a_{i_{1}} \otimes \cdots \otimes a_{i_{1}+\left|\phi_{1}\right|-1}\right) \otimes{ }^{g_{1}} a_{i_{1}+\left|\phi_{1}\right|} \otimes \cdots\right. \\
\cdots \otimes{ }^{g_{j-1} \cdots g_{1}} a_{i_{j}-1} \otimes \phi_{j}^{\circ}\left({ }^{g_{j-1} \cdots g_{1}} a_{i_{j}} \otimes \cdots\right) \otimes{ }^{g_{j} \cdots g_{1}} a_{i_{j}+\left|\phi_{j}\right|} \otimes \cdots \\
\left.\cdots \otimes \phi_{k}^{\circ}\left({ }^{g_{k-1} \cdots g_{1}} a_{i_{k}} \otimes \cdots\right) \otimes{ }^{g_{k} \cdots g_{1}} a_{i_{k}+\left|\phi_{j}\right|} \otimes \cdots\right) g g_{k} \cdots g_{1}, \quad
\end{gathered}
$$

where

$$
\mathcal{I}:=\left\{\boldsymbol{I}=\left(i_{1}<i_{2}<\cdots<i_{k}\right) \mid 0<i_{1} \text { and } \forall 1 \leqslant j<k, i_{j}+\left|\phi_{j}\right| \leqslant i_{j+1}\right\} .
$$

Here $\phi^{\circ} \in C^{\bullet}(A, A)$ such that $\phi=\phi^{\circ} g \in C^{\bullet}(A, A g)$. Similarly for $\phi_{i}^{\circ}$. We will set $\phi\left\{\phi_{1}, \phi_{2}, \cdots \phi_{k}\right\}=$ 0 , if $k>p$. This extends linearly to brace structures on $G$-twisted Hochschild cochains

$$
C^{\bullet}(A, A[G]) \rightarrow C^{\bullet}\left(C^{\bullet}(A, A[G]), C^{\bullet}(A, A[G])\right), \quad \phi \mapsto \phi\{\cdots\}
$$

which we call $G$-twisted braces.

Remark. We may use tree graphs to express terms above. For example, we write

$$
(-1)^{\left(i_{1}-1\right)\left(\left|\phi_{1}\right|-1\right)+\left(i_{2}-1\right)\left(\left|\phi_{2}\right|-1\right)}
$$

for

$$
\begin{gathered}
(-1)^{\left(i_{1}-1\right)\left(\left|\phi_{1}\right|-1\right)+\left(i_{2}-1\right)\left(\left|\phi_{2}\right|-1\right)} \phi^{\circ}\left(a_{1} \otimes \cdots \otimes a_{i_{1}-1} \otimes \phi_{1}^{\circ}\left(a_{i_{1}} \otimes \cdots\right) \otimes{ }^{g_{1}} a_{i_{1}+\left|\phi_{1}\right|} \otimes \cdots\right. \\
\left.\cdots \otimes{ }_{1}^{g} a_{i_{2}-1} \otimes \phi_{2}^{\circ}\left({ }^{g} a_{i_{2}} \otimes \cdots\right) \otimes{ }^{g_{2} g_{1}} a_{i_{2}+\left|\phi_{2}\right|} \otimes \cdots\right) g g_{2} g_{1},
\end{gathered}
$$

as a term in $\phi\left\{\phi_{1}, \phi_{2}\right\}\left(a_{1}, a_{2}, \cdots\right)$. As another example, the following graph

$$
(-1)^{\left(i_{1}-1\right)\left(\left|\phi_{1}\right|-1\right)+\left(i_{2}-1\right)\left(\left|\phi_{2}\right|-1\right)} g g_{1} g_{2}
$$

for

$$
\begin{gathered}
(-1)^{\left(i_{1}-1\right)\left(\left|\phi_{1}\right|-1\right)+\left(i_{2}-1\right)\left(\left|\phi_{2}\right|-1\right)} \phi^{\circ}\left(a_{1} \otimes \cdots \otimes a_{i_{1}-1} \otimes \phi_{1}^{\circ}\left(a_{i_{1}} \otimes \cdots\right) \otimes{ }^{g_{1}} a_{i_{1}+\left|\phi_{1}\right|} \otimes \cdots\right. \\
\left.\cdots \otimes{ }_{1}^{g} a_{i_{2}-1} \otimes{ }^{g_{1}} \phi_{2}^{\circ}\left(a_{i_{2}} \otimes \cdots\right) \otimes{ }^{g_{1} g_{2}} a_{i_{2}+\left|\phi_{2}\right|} \otimes \cdots\right) g g_{1} g_{2}
\end{gathered}
$$

appears as a term in $\phi\left\{\phi_{1}\right\}\left\{\phi_{2}\right\}\left(a_{1}, a_{2}, \cdots\right)$.

Remark. This brace structure is a generalization of twisted versions of Gerstenhaber products in [23, 43]. 
Proposition 2.2 ( $G$-twisted higher pre-Jacobi identities). Given $\phi \in C^{\bullet}(A, A g), \phi_{i} \in C^{\bullet}\left(A, A g_{i}\right)$ with $1 \leqslant i \leqslant n$ and $\psi_{j} \in C^{\bullet}\left(A, A h_{j}\right)$ with $1 \leqslant j \leqslant m$, we have the following $G$-twisted version of higher pre-Jacobi identities

$$
\begin{aligned}
& \phi\left\{\phi_{1}, \phi_{2} \cdots \phi_{n}\right\}\left\{\psi_{1}, \psi_{2} \cdots \psi_{m}\right\} \\
= & \sum_{j \in \mathcal{J}}(-1)^{\sum_{i=1}^{n} \xi_{i}\left(\left|\phi_{i}\right|-1\right)} \phi\left\{\psi_{1}, \cdots \psi_{j_{1}-1}, \phi_{1}\left\{\psi_{j_{1}}, \cdots \psi_{j_{1}^{\prime}-1}\right\}, g_{1}^{*}\left(\psi_{j_{1}^{\prime}}\right), \cdots\right. \\
& \cdots\left(g_{i-1} \cdots g_{1}\right)^{*}\left(\psi_{j_{i}-1}\right), \phi_{i}\left\{\left(g_{i-1} \cdots g_{1}\right)^{*}\left(\psi_{j_{i}}\right), \cdots\left(g_{i-1} \cdots g_{1}\right)^{*}\left(\psi_{j_{i}^{\prime}-1}\right)\right\}, \cdots \\
& \left.\cdots \phi_{n}\left\{\left(g_{n-1} \cdots g_{1}\right)^{*}\left(\psi_{j_{n}}\right), \cdots\left(g_{n-1} \cdots g_{1}\right)^{*}\left(\psi_{j_{n}-1}\right)\right\}, \cdots\left(g_{n} \cdots g_{1}\right)^{*}\left(\psi_{m}\right)\right\},
\end{aligned}
$$

where

$$
\mathcal{J}:=\left\{\boldsymbol{j}=\left(j_{1} \leqslant j_{1}^{\prime}<\cdots<j_{n} \leqslant j_{n}^{\prime}\right) \mid 1 \leqslant j_{1}, j_{n}^{\prime} \leqslant m \text { and } \forall 1 \leqslant i<n, j_{i}^{\prime} \leqslant\left|\phi_{i}\right|+j_{i}\right\},
$$

and

$$
\xi_{i}:=\left|\psi_{1}\right|+\left|\psi_{2}\right|+\cdots\left|\psi_{j_{i}-1}\right|-j_{i}+1
$$

Proof. We illustrate by an example when $n=m=2$. The left hand side of $(2.18)$ consists of terms like

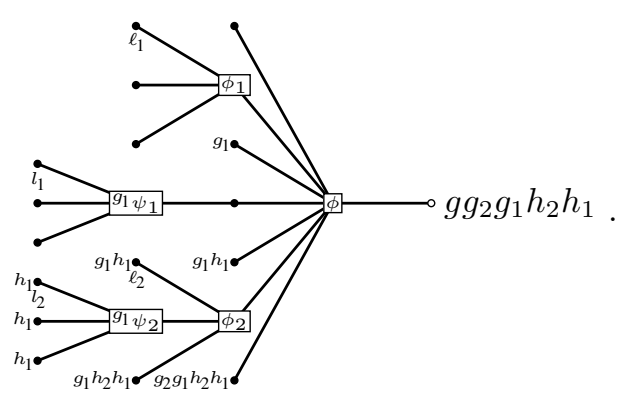

The right half side of 2.18) consists of terms like

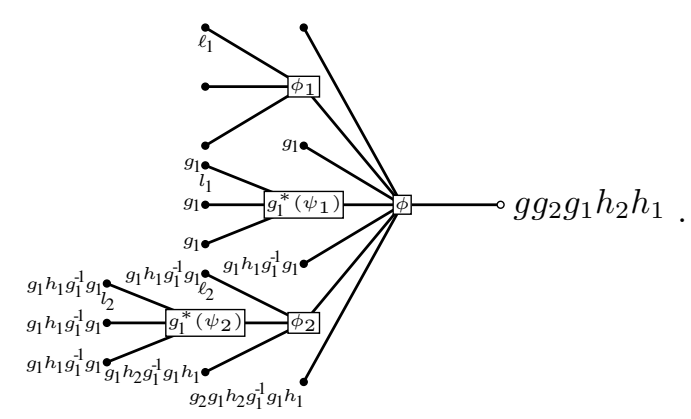

By 2.16), we see 2.18) hold.

Lemma 2.3. The twisted brace structures are G-equivariant with respect to the G-action defined in (2.16), i.e., $\forall h \in G$ and $\phi, \phi_{1}, \phi_{2}, \cdots \in C^{\bullet}(A, A[G])$, we have

$$
h^{*}\left(\phi\left\{\phi_{1}, \phi_{2}, \cdots\right\}\right)=h^{*}(\phi)\left\{h^{*}\left(\phi_{1}\right), h^{*}\left(\phi_{2}\right), \cdots\right\} .
$$


Proof. We illustrate for the case $\phi\left\{\phi_{1}, \phi_{2}\right\}$. Terms in both sides are given by

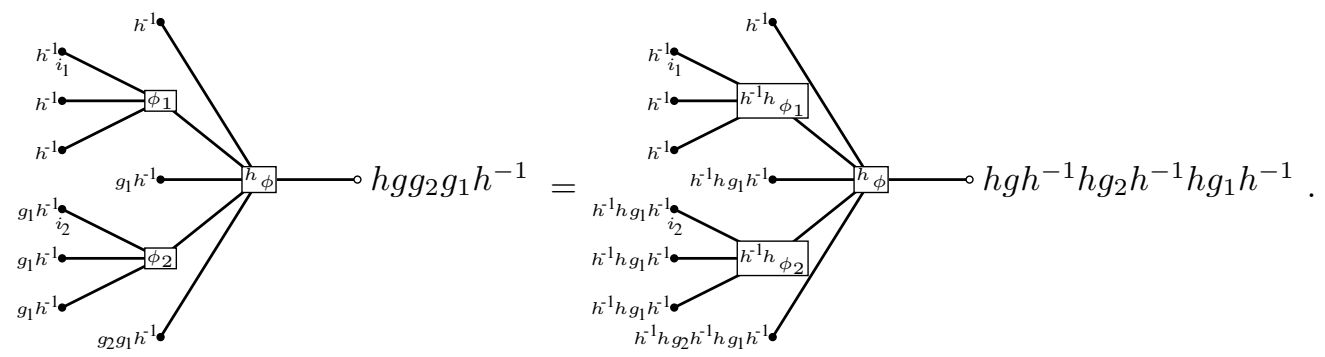

\section{$G$-twisted Hochschild differential and cup product}

Now we introduce differentials on $G$-twisted Hochschild cochains. We will always identify

$$
C^{\bullet}(A, A) \cong C^{\bullet}(A, A \mathrm{e}) \hookrightarrow C^{\bullet}(A, A[G])
$$

as the identity sector of $C^{\bullet}(A, A[G])$. In particular, we identify the product and the curving of $A$

$$
m_{2} \in C^{2}(A, A \mathrm{e}), \quad m_{0} \in C^{0}(A, A \mathrm{e}),
$$

as $G$-invariant cochains in $C^{\bullet}(A, A[G])$.

Definition 2.11. We define the Hochschild differential $\partial_{H}$ and the curving differential $\mathrm{d}_{W}$ on $G$-twisted Hochschild cochains by

$$
\partial_{H}(\phi)=(-1)^{|\phi|-1} m_{2}\{\phi\}-\phi\left\{m_{2}\right\}, \quad \mathrm{d}_{W}(\phi)=\phi\left\{m_{0}\right\}, \quad \phi \in C^{\bullet}(A, A[G]) .
$$

We also denote

$$
\partial_{H}^{W}=\partial_{H}+\mathrm{d}_{W} .
$$

Definition 2.12. We define the cup product on $G$-twisted Hochschild cochains by

$$
\phi_{1} \cup \phi_{2}=(-1)^{\left|\phi_{1}\right|\left(\left|\phi_{2}\right|-1\right)} m_{2}\left\{\phi_{1}, g_{1}^{*} \phi_{2}\right\}, \quad \phi_{i} \in C^{\bullet}\left(A, A g_{i}\right) .
$$

Note that this is the usual cup product that arises from the algebra structure on $A[G]$ by Definition 2.10 ,

Lemma 2.4. The following G-twisted version of higher homotopy identities holds,

$$
\begin{aligned}
& \partial_{H}(\phi)\left\{\phi_{1}, \phi_{2}, \cdots, \phi_{n}\right\} \\
= & (-1)^{\xi_{n}} \partial_{H}\left(\phi\left\{\phi_{1}, \cdots, \phi_{n}\right\}\right)-\sum_{k=1}^{n}(-1)^{\xi_{k}} \phi\left\{\phi_{1}, \cdots, \phi_{k-1}, \partial_{H}\left(\phi_{k}\right), \phi_{k+1}, \cdots, \phi_{n}\right\} \\
& +(-1)^{\left|\phi_{1}\right| \xi_{1}^{\prime}} \phi_{1} \cup\left(g_{1}^{-1}\right)^{*} \phi\left\{\phi_{2}, \phi_{3}, \cdots, \phi_{n}\right\} \\
& +\sum_{k=1}^{n-1}(-1)^{\xi_{k}+\left|\phi_{k}\right|\left|\phi_{k+1}\right|} \phi\left\{\phi_{1}, \cdots, \phi_{k} \cup\left(g_{i}^{-1}\right)^{*} \phi_{k+1}, \cdots, \phi_{n}\right\} \\
& -(-1)^{|\phi|\left|\phi_{n}\right|+\xi_{n-1} \xi_{n-1}^{\prime}} \phi\left\{\phi_{1}, \phi_{2}, \cdots, \phi_{n-1}\right\} \cup\left(\left(g_{n-1} \cdots g_{1}\right)^{-1}\right)^{*} \phi_{n},
\end{aligned}
$$

with

$$
\left\{\begin{array}{l}
\xi_{k}:=\left|\phi_{1}\right|+\left|\phi_{2}\right|+\cdots+\left|\phi_{k}\right|-k, \\
\xi_{k}^{\prime}:=\left|\phi_{k+1}\right|+\left|\phi_{k+2}\right|+\cdots+\left|\phi_{n}\right|-(n-k) .
\end{array}\right.
$$

Here $\phi \in C^{\bullet}(A, A g), \phi_{i} \in C^{\bullet}\left(A, A g_{i}\right)$. The same is true if $\partial_{H}$ is replaced by $\partial_{H}^{W}$. 
Proof. The lemma follows from the twisted higher pre-Jacobi identities and the $G$-invariance of $m_{2}, m_{0}$.

Corollary 2.5. The cup product $\cup$ on $C^{\bullet}(A, A[G])$ satisfies the following twisted commutativity up to homotopy: for any $\phi \in C^{\bullet}(A, A g), \phi_{1} \in C^{\bullet}\left(A, A g_{1}\right)$

$$
(-1)^{\left|\phi_{1}\right|} \partial_{H}\left(\phi\left\{\phi_{1}\right\}\right)+\left(\partial_{H} \phi\right)\left\{\phi_{1}\right\}-(-1)^{\left|\phi_{1}\right|} \phi\left\{\partial_{H} \phi_{1}\right\}=\phi_{1} \cup\left(g_{1}^{-1}\right)^{*} \phi-(-1)^{|\phi|\left|\phi_{1}\right|} \phi \cup \phi_{1} .
$$

Proof. This follows from Lemma 2.4 in the case $n=1$.

Corollary 2.6. The triple $\left\{C^{\bullet}(A, A[G]), \cup, \partial_{H}\right\}$ defines a differential graded algebra. If we replace $\partial_{H}$ with $\partial_{H}^{W}$, we get a $\mathbb{Z} / 2 \mathbb{Z}$-graded differential graded algebra.

Proof. Let $\phi \in C^{\bullet}(A, A g), \phi_{i} \in C^{\bullet}\left(A, A g_{i}\right)$. Lemma2.4 implies

$$
\begin{aligned}
& \partial_{H}\left(\phi\left\{\phi_{1}, \phi_{2}\right\}\right)-(-1)^{\left|\phi_{2}\right|-1} \phi\left\{\partial_{H} \phi_{1}, \phi_{2}\right\}-\phi\left\{\phi_{1}, \partial_{H} \phi_{2}\right\}-(-1)^{\left|\phi_{1}\right|+\left|\phi_{2}\right|} \partial_{H}(\phi)\left\{\phi_{1}, \phi_{2}\right\} \\
= & (-1)^{\left(\left|\phi_{1}\right|-1\right)\left|\phi_{2}\right|}\left(\phi\left\{\phi_{1} \cup\left(g_{1}^{-1}\right)^{*} \phi_{2}\right\}-(-1)^{(|\phi|-1)\left|\phi_{2}\right|} \phi\left\{\phi_{1}\right\} \cup\left(g_{1}^{-1}\right)^{*} \phi_{2}-\phi_{1} \cup\left(g_{1}^{-1}\right)^{*} \phi\left\{\phi_{2}\right\}\right) .
\end{aligned}
$$

Set $\phi=m_{2}$ and use $m_{2}\left\{m_{2}\right\}=0$, we find

$$
\partial_{H}\left(\phi_{1} \cup \phi_{2}\right)=\partial_{H}\left(\phi_{1}\right) \cup \phi_{2}+(-1)^{\left|\phi_{1}\right|} \phi_{1} \cup \partial_{H}\left(\phi_{2}\right) .
$$

The proof for $\partial_{H}^{W}$ is similar.

Definition 2.13. Let

$$
\mathrm{HH}(A, A[G])=\mathrm{H}\left(C^{\bullet}(A, A[G]), \partial_{H}\right), \quad \mathrm{HH}_{c}(A, A[G])=\mathrm{H}\left(C_{c}^{\bullet}(A, A[G]), \partial_{H}\right)
$$

denote the $G$-twisted Hochschild cohomologies and similarly for the curved case

$\mathrm{HH}\left(A_{W}, A_{W}[G]\right)=\mathrm{H}\left(C^{\bullet}(A, A[G]), \partial_{H}+\mathrm{d}_{W}\right), \quad \mathrm{HH}_{c}\left(A_{W}, A_{W}[G]\right)=\mathrm{H}\left(C_{c}^{\bullet}(A, A[G]), \partial_{H}+\mathrm{d}_{W}\right)$.

All the above cohomologies carry a natural $G$-action induced by (2.16).

Theorem 2.7. The cup product $\cup$ defines $\mathbb{Z}$-graded algebras on $\mathrm{HH}(A, A[G]), \mathrm{HH}_{c}(A, A[G])$ and $\mathbb{Z} / 2 \mathbb{Z}$ graded algebras on $\mathrm{HH}\left(A_{W}, A_{W}[G]\right), \mathrm{HH}_{c}\left(A_{W}, A_{W}[G]\right)$ satisfying the twisted commutativity relation

$$
\left[\phi_{1}\right] \cup\left[\phi_{2}\right]=(-1)^{\left|\phi_{1}\right|\left|\phi_{2}\right|}\left[\phi_{2}\right] \cup\left(g_{2}^{-1}\right)^{*}\left[\phi_{1}\right], \quad \phi_{i} \in C^{\bullet}\left(A, A g_{i}\right) .
$$

Moreover, their $G$-invariant subspaces, $\mathrm{HH}(A, A[G])^{G}, \mathrm{HH}_{c}(A, A[G])^{G}, \mathrm{HH}\left(A_{W}, A_{W}[G]\right)^{G}$ and $\mathrm{HH}_{c}\left(A_{W}, A_{W}[G]\right)^{G}$, inherit natural Gerstenhaber algebra structures.

Proof. The induced cup product on cohomologies and twisted commutativity follow from 2.21) 2.22). To see Gerstenhaber algebra structures on $G$-invariant cohomologies, we consider for example

$$
\mathrm{HH}(A, A[G])^{G}=\mathrm{H}\left(C^{\bullet}(A, A[G])^{G}, \partial_{H}\right),
$$

where $C^{\bullet}(A, A[G])^{G}$ are $G$-invariant cochains. On $C^{\bullet}(A, A[G])^{G}$, the $G$-twisted higher pre-Jacobi identities (2.18) reduce to the same form as the untwisted one (2.7), from which we deduce the Gerstenhaber algebra structures by (2.5) 2.8) 2.9) (2.10). 
Remark. The $G$-twisted commutativity of the cup product is also obtained by Shklyarov in [46].

Proposition 2.8. Let $H$ be a subgroup of $G$, then the inclusion

$$
C^{\bullet}(A, A[H]) \hookrightarrow C^{\bullet}(A, A[G])
$$

induce an embedding of $\mathbb{Z} / 2 \mathbb{Z}$-graded algebras

$$
\mathrm{HH}_{c}\left(A_{W}, A_{W}[H]\right) \hookrightarrow \mathrm{HH}_{c}\left(A_{W}, A_{W}[G]\right) .
$$

The same is true if we consider $\mathrm{HH}(A, A[H]), \mathrm{HH}\left(A_{W}, A_{W}[H]\right)$.

Proof. It is easy to see that $\mathrm{HH}_{c}\left(A_{W}, A_{W}[H]\right)$ is a $\mathbb{Z} / 2 \mathbb{Z}$-graded subspace of $\mathrm{HH}_{c}\left(A_{W}, A_{W}[G]\right)$ and closed under the cup product.

\subsection{A comparison between Gerstenhaber algebras}

In this subsection, $G$ will be a finite group. In this case, Ştefan [48] proved that there is an isomorphism as graded vector spaces between $\mathrm{HH}^{\bullet}(A[G])$ and $\mathrm{HH}^{\bullet}(A, A[G])^{G}$ (see also [11]). Using $G$-twisted brace structures, we extend this to an isomorphism between Gerstenhaber algebras.

Consider the following map [2, 23, 43, 6]

$$
\begin{aligned}
\Psi: C^{\bullet}(A, A[G])^{G} & \rightarrow C^{\bullet}(A[G], A[G]), \\
\phi & \mapsto \Psi(\phi),
\end{aligned}
$$

where for $a_{1} g_{1}, a_{2} g_{2}, \cdots a_{p} g_{p} \in A[G]$,

$$
\Psi(\phi)\left(a_{1} g_{1} \otimes \cdots a_{2} g_{2} \otimes \cdots \otimes a_{p} g_{p}\right)=\phi\left(a_{1} \otimes{ }^{g_{1}} a_{2} \otimes \cdots \otimes{ }^{g_{1} g_{2} \cdots g_{p-1}} a_{p}\right) g_{1} g_{2} \cdots g_{p} .
$$

Lemma 2.9. $\Psi$ preserves brace structures: for any $\phi, \phi_{i} \in C^{\bullet}(A, A[G])^{G}$,

$$
\Psi(\phi)\left\{\Psi\left(\phi_{1}\right), \Psi\left(\phi_{2}\right), \cdots\right\}=\Psi\left(\phi\left\{\phi_{1}, \phi_{2}, \cdots\right\}\right) .
$$

Proof. Let $\phi_{k}$ be a $G$-invariant $G$-twisted cochain. We write

$$
\phi_{k}=\sum_{g \in G} \phi_{k}^{g} g, \quad \phi_{k}^{g} \in C^{\bullet}(A, A) .
$$

$G$-invariance implies that

$$
h^{*}\left(\phi_{k}^{g}\right)=\phi_{k}^{h g h^{-1}} .
$$

Given input $a_{1} h_{1} \otimes a_{2} h_{2} \otimes \cdots$, the left half side of (2.24) gives a sum of terms like

$$
\begin{aligned}
& \pm \phi^{g}\left(a_{1} \otimes{ }^{h_{1}} a_{2} \otimes \cdots \otimes{ }^{\hbar_{i_{1}}} \phi_{1}^{\hbar_{1_{1}}^{-1} g_{1} \hbar_{i_{1}}}\left(a_{i_{1}} \otimes{ }^{h_{i_{1}}} a_{i_{1}+1} \otimes \cdots\right) \otimes \cdots\right. \\
& \left.\ldots g_{k-1} g_{k-2} \cdots g_{1} \hbar_{i_{k}} \phi_{k}^{\left(g_{k-1} \cdots g_{1} \hbar_{i_{k}}\right)^{-1} g_{k} g_{k-1} \cdots g_{1} \hbar_{i_{k}}}\left(a_{i_{k}} \otimes \cdots\right) \otimes \cdots\right) g \cdots g_{2} g_{1} h_{1} h_{2} \cdots \\
& = \pm \phi^{g}\left(a_{1} \otimes{ }^{h_{1}} a_{2} \otimes \cdots \otimes \phi_{1}^{g_{1}}\left({ }^{\hbar_{i_{1}}} a_{i_{1}} \otimes{ }^{\hbar_{i_{1}} h_{i_{1}}} a_{i_{1}+1} \otimes \cdots\right) \otimes \cdots\right. \\
& \left.\cdots \phi_{k}^{g_{k}}\left(g_{k-1} g_{k-2} \cdots g_{1} \hbar_{i_{k}} a_{i_{k}} \otimes \cdots\right) \otimes \cdots\right) g \cdots g_{2} g_{1} h_{1} h_{2} \cdots,
\end{aligned}
$$


where $\hbar_{i}:=h_{1} h_{2} \cdots h_{i}$. The right half side of (2.24) gives a sum of terms like

$$
\begin{aligned}
& \pm \phi^{g}\left(a_{1} \otimes{ }^{h_{1}} a_{2} \otimes \cdots \otimes \phi_{1}^{g_{1}}\left({ }^{\hbar_{i_{1}}} a_{i_{1}} \otimes{ }^{\hbar_{i_{1}} h_{i_{1}}} a_{i_{1}+1} \otimes \cdots\right) \otimes \cdots\right. \\
& \left.\cdots \phi_{k}^{g_{k}}\left(g_{k-1} g_{k-2} \cdots g_{1} \hbar_{i_{k}} a_{i_{k}} \otimes \cdots\right) \otimes \cdots\right) g \cdots g_{2} g_{1} h_{1} h_{2} \cdots .
\end{aligned}
$$

The Lemma follows.

Theorem 2.10. Let $G$ be a finite group. Then $\Psi$ defined by (2.23) induces an isomorphism between $\mathbb{Z}$-graded Gerstenhaber algebras

$$
\Psi: \mathrm{HH}^{\bullet}(A, A[G])^{G} \rightarrow \mathrm{HH}^{\bullet}(A[G]) .
$$

Proof. It is easy to see that $\Psi$ is compatible with Hochschild differential $\partial_{H}$. By [2], $\Psi$ induces a $\mathbb{Z}$-graded vector space isomorphism between $\mathrm{HH}^{\bullet}(A, A[G])^{G}$ and $\mathrm{HH}^{\bullet}(A[G])$. The theorem is now a formal consequence of Lemma 2.9 .

Now we consider the curved case.

Theorem 2.11. Let $G$ be a finite group, $(A, W, G)$ be a $G$-twisted curved algebra. Then $\Psi$ defined by (2.23) induces an isomorphism between $\mathbb{Z} / 2 \mathbb{Z}$-graded Gerstenhaber algebras

$$
\Psi: \mathrm{HH}_{c}\left(A_{W}, A_{W}[G]\right)^{G} \rightarrow \mathrm{HH}_{c}\left(A_{W}[G]\right) .
$$

Proof. By Lemma 2.9, we only need to prove that

$$
\Psi: C_{c}^{\bullet}(A, A[G])^{G} \rightarrow C_{c}^{\bullet}(A[G])
$$

induce a vector space isomorphism on $\left(\partial_{H}+\mathrm{d}_{W}\right)$-cohomology. Introduce a formal variable $u$ of degree 2 and we extend $\Psi$ to a cochain map between $\mathbb{Z}$-graded complex

$$
\Psi:\left(C_{c}^{\bullet}(A, A[G])^{G}\left[u, u^{-1}\right], \partial_{H}+u \mathrm{~d}_{W}\right) \rightarrow\left(C_{c}^{\bullet}(A[G], A[G])\left[u, u^{-1}\right], \partial_{H}+u \mathrm{~d}_{W}\right) .
$$

For any $k \in \mathbb{Z}$, there are natural identifications

$$
\begin{aligned}
& \mathrm{HH}_{c}^{\overline{0}}\left(A_{W}, A_{W}[G]\right)^{G}=\mathrm{H}^{2 k}\left(C_{c}^{\bullet}(A, A[G])^{G}[u], \partial_{H}+u \mathrm{~d}_{W}\right) \\
& \mathrm{HH}_{c}^{\overline{1}}\left(A_{W}, A_{W}[G]\right)^{G}=\mathrm{H}^{2 k+1}\left(C_{c}^{\bullet}(A, A[G])^{G}[u], \partial_{H}+u \mathrm{~d}_{W}\right)
\end{aligned}
$$

and similarly for $\mathrm{HH}_{c}\left(A_{W}[G]\right)$. It suffices to show that $(2.25)$ is a quasi-isomorphism.

Consider the decreasing filtration $\cdots \subset F^{p+1} \subset F^{p} \subset F^{p-1} \subset \cdots$ where

$$
F^{p}=u^{p} C_{c}^{\bullet}[u] .
$$

Here $C_{c}^{\bullet}$ denotes $C_{c}^{\bullet}(A, A[G])^{G}$ or $C_{c}^{\bullet}(A[G], A[G])$. Since we work with compact type complex, this filtration is exhaustive (see [53] for details) and there is an associated convergent spectral sequence. $\Psi$ in (2.25) induces an isomorphism between $E_{1}$-pages which are computed by $\partial_{H}$-cohomologies. It follows that (2.25) defines a quasi-isomorphism. 


\section{Orbifold Landau-Ginzburg B-models}

In this section we study the orbifold Landau-Ginzburg model associated to a $G$-twisted curved algebra

$$
\left(A=\mathbb{C}\left[x_{1}, \cdots, x_{N}\right], W, G\right),
$$

where $W$ is a weighted homogeneous invertible polynomial and $G$ is a subgroup of $\left(\mathbb{C}^{*}\right)^{N}$ preserving $W$. We show that the cohomology $\mathrm{HH}_{c}\left(A_{W}, A_{W}[G]\right)$ is a $G$-Frobenius algebra. In particular, the $G$-invariant subspace $\mathrm{HH}_{c}\left(A_{W}, A_{W}[G]\right)^{G}$ has an induced Frobenius algebra structure. We give closed formulae for the cup product on $\mathrm{HH}_{c}\left(A_{W}, A_{W}[G]\right)^{G}$ for all Fermat, Loop and Chain types. This is computed via an explicit homotopy between Koszul resolution and bar resolution.

For convenience, we fix the following notations in this section.

Definition 3.1. For $g \in G$, we let

$$
\operatorname{Fix}(g)=\left\{v \in \mathbb{C}^{N} \mid{ }^{g} v=v\right\} \subset \mathbb{C}^{N}
$$

be the fixed locus of $g$ and

$$
A_{g}=\mathbb{C}[\operatorname{Fix}(g)]
$$

denote polynomial functions on $\operatorname{Fix}(g)$. We write

$$
N_{g}=\operatorname{dim}_{\mathbb{C}} \operatorname{Fix}(g), \quad W_{g}=\left.W\right|_{\mathrm{Fix}(g)} \in A_{g} .
$$

We also denote $\boldsymbol{I}_{g}=\left\{i_{1}<i_{2}<\cdots<i_{N-N_{g}}\right\}$ where $\left\{x_{i_{k}}\right\}$ 's are variables such that ${ }^{g} x_{i_{k}} \neq x_{i_{k}} . \boldsymbol{I}_{g}$ will be called the moving index of $g$.

Definition 3.2. Let $x_{1}^{\gamma_{1}} \cdots x_{N}^{\gamma_{N}}$ be a monomial in $A$, and $g \in G$. We define

$$
\rho_{i}(g)\left(x_{1}^{\gamma_{1}} \cdots x_{N}^{\gamma_{N}}\right)=\left({ }^{g} x_{1}\right)^{\gamma_{1}} \cdots\left({ }^{g} x_{i-1}\right)^{\gamma_{i-1}} x_{i}^{\gamma_{i}} \cdots x_{N}^{\gamma_{N}},
$$

and the quantum differential operator

$$
\partial_{x_{i}}^{g}\left(x_{1}^{\gamma_{1}} \cdots x_{N}^{\gamma_{N}}\right)= \begin{cases}{[\gamma]_{\lambda_{i}} x_{1}^{\gamma_{1}} \cdots x_{i}^{\gamma_{i}-1} \cdots x_{N}^{\gamma_{N}}} & \text { if } \gamma_{i}>0 \\ 0 & \text { else. }\end{cases}
$$

Here $\lambda_{i}$ is the weight: ${ }^{g} x_{i}=\lambda_{i} x_{i} \cdot[\gamma]_{\lambda}(\gamma \geqslant 1)$ is defined by

$$
[\gamma]_{\lambda}=1+\lambda+\lambda^{2}+\cdots+\lambda^{\gamma-1}
$$

Both $\rho_{i}(g)$ and $\partial_{x_{i}}^{g}$ extend linearly to operators on $A$.

\subsection{Bar resolution vs. Koszul resolution}

For simplicity, we work with the reduced bar resolution (or 'normalized bar resolution', see [34]),

$$
\cdots \stackrel{\mathrm{b}}{\longrightarrow} A \otimes \bar{A}^{\otimes 3} \otimes A \stackrel{\mathrm{b}}{\longrightarrow} A \otimes \bar{A}^{\otimes 2} \otimes A \stackrel{\mathrm{b}}{\longrightarrow} A \otimes \bar{A} \otimes A \stackrel{\mathrm{b}}{\longrightarrow} A \otimes A \stackrel{m_{2}}{\longrightarrow} A \longrightarrow 0,
$$

where $\bar{A}=A / \mathbb{C}=\mathbb{C}\left[x^{i}\right] / \mathbb{C}$. It gives a free resolution of $A$ as $A^{e}$-modules (equivalently $A$-bimodules) where $A^{e}=A \otimes A^{\text {op }}$. Applying the functor $\operatorname{Hom}_{A^{e}}(\cdot, A[G]$ ), we obtain the (reduced) G-twisted Hochschild cochain complex

$$
\partial_{H}: C^{p}(\bar{A}, A[G]) \rightarrow C^{p+1}(\bar{A}, A[G])
$$


Here $C^{p}(\bar{A}, A[G])=\operatorname{Hom}\left(\bar{A}^{\otimes p}, A[G]\right)$ and $\partial_{H}$ is the Hochschild differential which is also well-defined on the reduced complex. We will denote

$$
C^{\bullet}(\bar{A}, A[G])=\prod_{p=0}^{\infty} C^{p}(\bar{A}, A[G]), \quad C_{c}^{\bullet}(\bar{A}, A[G])=\bigoplus_{p=0}^{\infty} C^{p}(\bar{A}, A[G]) .
$$

The curving differential $d_{W}$ is defined the same as before

$$
\mathrm{d}_{W}: C^{p}(\bar{A}, A[G]) \rightarrow C^{p-1}(\bar{A}, A[G]) .
$$

Since $A=\mathbb{C}\left[x_{i}\right]$, we have a simpler $A^{e}$-module resolution of $A$ via the Koszul resolution

$$
\cdots \stackrel{\tilde{\mathrm{b}}}{\longrightarrow} \underset{i<j<k}{\bigoplus_{<}} e^{i} e^{j} e^{k} A^{e} \stackrel{\tilde{\mathrm{b}}}{\longrightarrow} \bigoplus_{i<j} e^{i} e^{j} A^{e} \stackrel{\tilde{\mathrm{b}}}{\longrightarrow} \underset{i}{\oplus} e^{i} A^{e} \stackrel{\tilde{\mathrm{b}}}{\longrightarrow} A^{e} \stackrel{m}{\longrightarrow} A \longrightarrow 0,
$$

where $e^{i}$,s are odd variables: $\operatorname{deg} e^{i}=-1$ and $e^{i} e^{j}=-e^{j} e^{i}$. The Koszul differential $\tilde{\mathrm{b}}$ is a derivation of the ring $A^{e}\left[e^{i}\right]$ generated by

$$
\tilde{\mathrm{b}}\left(e^{i}\right)=x_{i} \otimes 1-1 \otimes x_{i} \in A^{e} .
$$

Applying the functor $\operatorname{Hom}_{A^{e}}(\cdot, A[G])$, we get the $G$-twisted Koszul cochain complex

$$
\partial_{K}: K^{\bullet}(A, A[G])=A\left[e_{i}\right][G] \rightarrow K^{\bullet+1}(A, A[G]) .
$$

Here $e_{i}$ is the dual basis of $e^{i}$ such that $\operatorname{deg}\left(e_{i}\right)=1, e_{i} e_{j}=-e_{j} e_{i}$. The Koszul differential $\partial_{H}$ is

$$
\partial_{K}(\phi g)=\sum_{i=1}^{N}\left(x_{i}-{ }^{g} x_{i}\right) e_{i} \phi g, \quad \phi \in A\left[e_{i}\right], g \in G .
$$

Definition 3.3. We define the curving differential on $G$-twisted Koszul cochains

$$
\tilde{\mathrm{d}}_{W}: K^{p}(A, A[G]) \rightarrow K^{p-1}(A, A[G])
$$

by

$$
\tilde{\mathrm{d}}_{W}\left(a e_{i_{1}} \cdots e_{i_{p}} g\right)=\sum_{k=1}^{p}(-1)^{k-1} a \rho_{i_{k}}(g)\left(\partial_{x_{i_{k}}}^{g}(W)\right) e_{i_{1}} \cdots \widehat{e_{i_{k}}} \cdots e_{i_{p}} g .
$$

Here $a \in A, g \in G$ and $1 \leq i_{1}<\cdots<i_{p} \leq N$.

In [42], Shepler and Witherspoon introduced chain maps between these two resolutions,

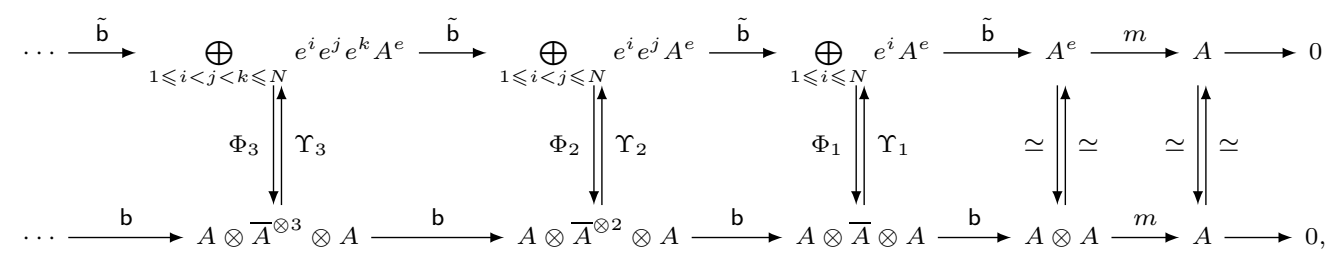

- The chain map $\Phi$ from Koszul resolution to bar resolution is given by

$$
\Phi_{p}: \quad e^{i_{1}} e^{i_{2}} \cdots e^{i_{p}} a \otimes b \mapsto \sum_{\sigma \in S_{p}}(-1)^{|\sigma|} a \otimes x_{i_{\sigma(1)}} \otimes \cdots \otimes x_{i_{\sigma(p)}} \otimes b . \quad a, b \in A .
$$


Let $\Phi^{*}$ denote the induced map on cochains

$$
\begin{aligned}
\Phi_{p}^{*}: C^{p}(\bar{A}, A[G]) & \rightarrow K^{p}(A, A[G]), \\
\phi & \mapsto \sum_{i_{1}<\cdots<i_{p}} \sum_{\sigma \in S_{p}}(-1)^{|\sigma|} \phi\left(x_{i_{\sigma(1)}} \otimes \cdots \otimes x_{i_{\sigma(p)}}\right) e_{i_{1}} \cdot e_{i_{p}} .
\end{aligned}
$$

- The chain map $\Upsilon$ from bar resolution to Koszul resolution is constructed in the following steps:

Step 1 Let $a \otimes a_{1} \otimes \cdots \otimes a_{p} \otimes b \in A \otimes \bar{A}^{\otimes p} \otimes A$ where $a_{k}=\prod_{i=1}^{N} x_{i}^{\gamma_{i}^{k}}\left(\gamma_{i}^{k} \geqslant 0\right)$.

Step 2 For each $1 \leqslant i_{1}<i_{2}<\cdots<i_{p} \leqslant N$ denoted by $\boldsymbol{I}$, let us define $\mathcal{S}(\boldsymbol{I})$ to be the set of sequence $s=\left(s_{1}, s_{2}, \cdots s_{p}\right)$ such that

$$
0 \leqslant s_{k}<\gamma_{i_{k}}^{k}
$$

Given $s \in \mathcal{S}(\boldsymbol{I})$, we define a splitting of each $a_{k}$ into two parts:

$$
\left\{\begin{array}{l}
a_{k, \boldsymbol{I}, \boldsymbol{s}}^{2}=x_{1}^{\gamma_{1}^{k} \cdots x_{\left(i_{k}-1\right)}^{\gamma_{\left(i_{k}-1\right)}^{k}} x_{i_{k}}^{s_{k}},} \\
a_{k, \boldsymbol{I}, \boldsymbol{s}}^{1}=x_{i_{k}}^{\gamma_{i_{k}}^{k}-1-s_{k}} x_{\left(i_{k}+1\right)}^{\gamma_{\left(i_{k}+1\right)}^{k}} \cdots x_{N}^{\gamma_{N}^{k}} .
\end{array}\right.
$$

Step $3 \Upsilon$ is defined by

$$
\Upsilon_{p}\left(a \otimes a_{1} \otimes \cdots \otimes a_{p} \otimes b\right)=\sum_{\boldsymbol{I}, \boldsymbol{s} \in \mathcal{S}(\boldsymbol{I})}\left(a a_{1}^{1} \cdots a_{p}^{1}\right) \otimes\left(a_{p}^{2} \cdots a_{1}^{2} b\right) e^{i_{1}} e^{i_{2}} \cdots e^{i_{p}},
$$

where $a_{k}^{1}$ and $a_{k}^{2}$ are short for $a_{k, \boldsymbol{I}, \boldsymbol{s}}^{1}$ and $a_{k, \boldsymbol{I}, \boldsymbol{s}}^{2}$. The proof for $\Upsilon$ being a chain map can be found in the Appendix of [42].

Let $\Upsilon^{*}$ denote the induced map on cochains

$$
\Upsilon_{p}^{*}: K^{p}(A, A[G]) \rightarrow C^{p}(\bar{A}, A[G]) .
$$

For $\psi=a e_{i_{1}} \cdots e_{i_{p}} g$ where $1 \leqslant i_{1}<i_{2}<\cdots<i_{p} \leqslant N$ denoted by $\boldsymbol{I}, a \in A, g \in G$, we have

$$
\begin{aligned}
\Upsilon_{p}^{*}(\psi)\left(a_{1} \otimes \cdots \otimes a_{p}\right) & =\psi\left(\Upsilon\left(1 \otimes a_{1} \otimes \cdots \otimes a_{p} \otimes 1\right)\right) \\
& =\sum_{\boldsymbol{s} \in \mathcal{S}(\boldsymbol{I})} a_{1}^{1} a_{2}^{1} \cdots a_{p}^{1} a\left({ }^{g} a_{1}^{2}\right)\left({ }^{g} a_{2}^{2}\right) \cdots\left({ }^{g} a_{p}^{2}\right) g \\
& =\rho_{i_{1}}(g)\left(\partial_{x_{i_{1}}}^{g} a_{1}\right) \rho_{i_{2}}(g)\left(\partial_{x_{i_{2}}}^{g} a_{2}\right) \cdots \rho_{i_{p}}(g)\left(\partial_{x_{i_{p}}}^{g} a_{p}\right) a g .
\end{aligned}
$$

Lemma 3.1. $\Upsilon^{*}$ is compatible with the curving differential

$$
\mathrm{d}_{W} \circ \Upsilon^{*}=\Upsilon^{*} \circ \tilde{\mathrm{d}}_{W} .
$$

Proof. It can be checked directly.

Theorem 3.2 ([43]). The composition

$$
\Upsilon \circ \Phi=\mathrm{id}
$$

is the identity on Koszul resolution. 
This theorem implies that the other composition $\Phi \circ \Upsilon$ will be homotopic to the identity id on bar resolution. Let us describe such a homotopy $\mathrm{H}$ explicitly. It will allow us to compute various algebraic structures on Hochschild cohomology and orbifold Landau-Ginzburg models.

Definition 3.4. We define $\mathrm{H}_{p}: A \otimes \bar{A}^{\otimes p} \otimes A \rightarrow A \otimes \bar{A}^{\otimes(p+1)} \otimes A$ by

$$
\begin{aligned}
& \mathrm{H}_{p}\left(a_{0} \otimes a_{1} \otimes \cdots \otimes a_{p} \otimes a_{p+1}\right) \\
= & \sum_{i=1}^{p+1}(-1)^{i} a_{0} \otimes \cdots \otimes a_{i-1} \otimes \Phi_{p-i+1} \circ \Upsilon_{p-i+1}\left(1 \otimes a_{i} \otimes \cdots \otimes a_{p} \otimes a_{p+1}\right) .
\end{aligned}
$$

Proposition 3.3. $\mathrm{H}$ gives a homotopy between id and $\Phi \circ \Upsilon$ on the bar resolution

$$
\mathrm{id}-\Phi \circ \Upsilon=\mathrm{b} \circ \mathrm{H}+\mathrm{H} \circ \mathrm{b} .
$$

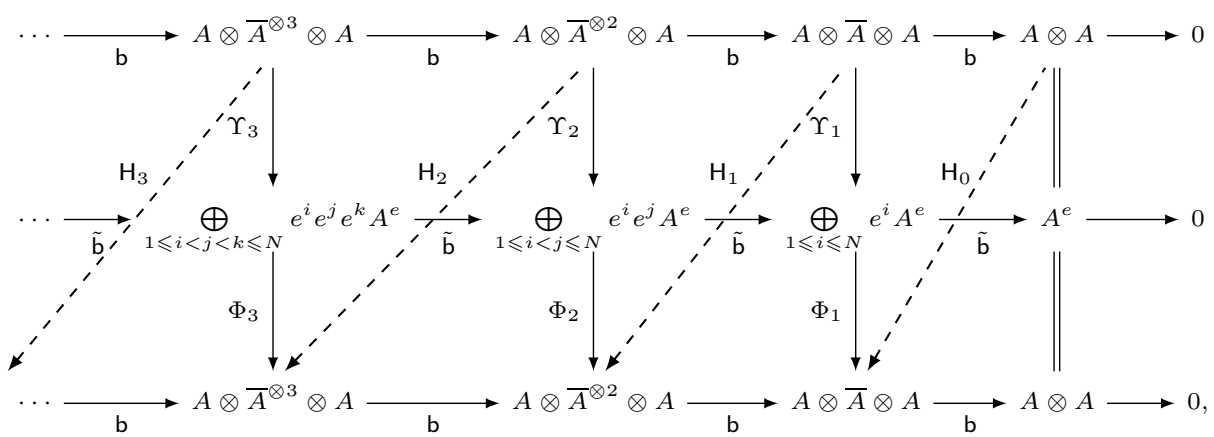

Dually, $\mathrm{H}$ induces a homotopy $\mathrm{H}^{*}$ between $\mathrm{id}$ and $\Upsilon^{*} \circ \Phi^{*}$ on $G$-twisted cochains

$$
\mathrm{id}-\Upsilon^{*} \circ \Phi^{*}=\mathrm{H}^{*} \circ \partial_{H}+\partial_{H} \circ \mathrm{H}^{*}: C^{\bullet}(\bar{A}, A[G]) \rightarrow C^{\bullet}(\bar{A}, A[G]) .
$$

Proof. Let $s$ be the homotopy for bar resolution,

$$
\cdots \underset{s}{\stackrel{\mathrm{b}}{\rightleftarrows}} A \otimes \bar{A}^{\otimes 3} \otimes A \underset{s}{\stackrel{\mathrm{b}}{\rightleftarrows}} A \otimes \bar{A}^{\otimes 2} \otimes A \underset{s}{\stackrel{\mathrm{b}}{\rightleftarrows}} A \otimes \bar{A} \otimes A \underset{s}{\stackrel{\mathrm{b}}{\rightleftarrows}} A \otimes A \underset{s}{\stackrel{m_{2}}{\rightleftarrows}} A \longrightarrow 0,
$$

where $s\left(a_{0} \otimes \cdots \otimes a_{k}\right)=1 \otimes a_{0} \otimes \cdots \otimes a_{k}$. It satisfies

$$
\mathrm{b} \circ s+s \circ \mathrm{b}=\mathrm{id} \text {. }
$$

Using the fact that $\Phi \circ \Upsilon$ commutes with $b$, we have

$$
\begin{aligned}
& (\mathrm{b} \circ \mathrm{H}+\mathrm{H} \circ \mathrm{b})\left(a_{0} \otimes a_{1} \otimes \cdots \otimes a_{p} \otimes a_{p+1}\right) \\
= & -\sum_{i=1}^{p+1} a_{0} \otimes \cdots \otimes\left(a_{i-1} \Phi \circ \Upsilon\left(1 \otimes a_{i} \otimes \cdots \otimes a_{p} \otimes a_{p+1}\right)\right) \\
& +a_{0} \otimes \cdots \otimes a_{p} \otimes m_{2}\left(\Phi \circ \Upsilon\left(1 \otimes a_{p+1}\right)\right)+\sum_{i=1}^{p} a_{0} \otimes \cdots \otimes \Phi \circ \Upsilon\left(a_{i} \otimes \cdots \otimes a_{p+1}\right) \\
= & (\mathrm{id}-\Phi \circ \Upsilon)\left(a_{0} \otimes a_{1} \otimes \cdots \otimes a_{p} \otimes a_{p+1}\right) .
\end{aligned}
$$

The last statement follows from the fact that $\mathrm{H}$ is an $A$-bimodule homomorphism. 
Therefore we have a homotopy retraction between two cochains

$$
\mathrm{H}^{*}\left(C^{\bullet}(\bar{A}, A[G]), \partial_{H}\right) \underset{\Upsilon^{*}}{\stackrel{\Phi^{*}}{\rightleftarrows}}\left(K^{\bullet}(A, A[G]), \partial_{K}\right) .
$$

Lemma 3.4. The homotopy $\mathrm{H}^{*}$ satisfies

$$
\mathrm{H}^{*} \circ \Upsilon^{*}=0, \quad \Phi^{*} \circ \mathrm{H}^{*}=0, \text { and } \mathrm{H}^{*} \circ \mathrm{H}^{*}=0 .
$$

In other words, the triple $\left(\Upsilon^{*}, \Phi^{*}, \mathrm{H}^{*}\right)$ is a special homotopy retraction (see for example [10]).

Proof. We have

$$
\begin{aligned}
& \Upsilon_{p+1} \circ \mathrm{H}_{p}\left(1 \otimes a_{1} \otimes \cdots \otimes a_{p} \otimes 1\right) \\
= & \sum_{i=1}^{p+1}(-1)^{i} \Upsilon_{p+1}\left(1 \otimes \cdots \otimes a_{i-1} \otimes \Phi_{p-i+1} \circ \Upsilon_{p-i+1}\left(1 \otimes a_{i} \otimes \cdots \otimes a_{p} \otimes 1\right)\right) \\
= & 0,
\end{aligned}
$$

where the last equality follows from (3.8) and the ordering (3.10).

$$
\begin{aligned}
& \mathrm{H} \circ \Phi\left(1 \otimes 1 e^{i_{1}} e^{i_{2}} \cdots e^{i_{p}}\right) \\
= & \sum_{\sigma \in S_{p}} \sum_{i=1}^{p+1}(-1)^{i+|\sigma|} 1 \otimes \cdots \otimes x_{\sigma(i-1)} \otimes \Phi_{p-i+1} \circ \Upsilon_{p-i+1}\left(1 \otimes x_{\sigma(i)} \otimes \cdots \otimes x_{\sigma(p)} \otimes 1\right),
\end{aligned}
$$

which is zero as a reduced chain since $\Phi_{p-i+1} \circ \Upsilon_{p-i+1}\left(1 \otimes x_{\sigma(i)} \otimes \cdots \otimes x_{\sigma(p)} \otimes 1\right)$ contributes 1 at some middle position. Similarly,

$$
\begin{aligned}
& \mathrm{H} \circ \mathrm{H}\left(1 \otimes a_{1} \otimes \cdots \otimes a_{p} \otimes 1\right) \\
= & \sum_{i=1}^{p+1}(-1)^{i} \mathrm{H}\left(1 \otimes \cdots \otimes a_{i-1} \otimes \Phi_{p-i+1} \circ \Upsilon_{p-i+1}\left(1 \otimes a_{i} \otimes \cdots \otimes a_{p} \otimes 1\right)\right)
\end{aligned}
$$

is zero as a reduced chain.

We will be interested in studying the $\left(\partial_{H}+\mathrm{d}_{W}\right)$-cohomology in terms of Koszul complex. Viewing $\mathrm{d}_{W}$ as a small perturbation, standard homological perturbation lemma (together with Lemma 3.1 and Lemma 3.4) allows us to construct a new homotopy retract $\left(\Upsilon^{*}, \tilde{\Phi}^{*}, \tilde{H}^{*}\right)$

$$
\tilde{\mathrm{H}}^{*} \bigodot\left(C \cdot(\bar{A}, A[G]), \partial_{H}+\mathrm{d}_{W}\right) \underset{\Upsilon^{*}}{\stackrel{\tilde{\Phi}^{*}}{\rightleftarrows}}\left(K^{\bullet}(A, A[G]), \partial_{K}+\tilde{\mathrm{d}}_{W}\right) .
$$

Here the perturbed homotopy $\tilde{\mathrm{H}}^{*}$ and retract $\tilde{\Phi}^{*}$ are defined by

$$
\tilde{\Phi}^{*}=\Phi^{*}-\Phi^{*}\left(\text { id }+\mathrm{d}_{W} \mathrm{H}^{*}\right)^{-1} \mathrm{~d}_{W} \mathrm{H}^{*}, \quad \tilde{\mathrm{H}}^{*}=\mathrm{H}^{*}\left(\mathrm{id}+\mathrm{d}_{W} \mathrm{H}^{*}\right)^{-1},
$$

where (id $\left.+\mathrm{d}_{W} \mathrm{H}^{*}\right)^{-1}=\sum_{m \geq 0}(-1)^{m}\left(\mathrm{~d}_{W} \mathrm{H}^{*}\right)^{m}$. It can be checked directly that

$$
\left\{\begin{array}{l}
\tilde{\Phi}^{*} \circ \Upsilon^{*}=\mathrm{id} \\
\text { id }-\Upsilon^{*} \circ \tilde{\Phi}^{*}=\tilde{\mathbf{H}}^{*} \circ\left(\partial_{H}+\mathrm{d}_{W}\right)+\left(\partial_{H}+\mathrm{d}_{W}\right) \circ \tilde{\mathbf{H}}^{*}
\end{array}\right.
$$

We will need the following property to compute the cup product $\cup$. 
Lemma 3.5. For any $\phi \in C^{p}(\bar{A}, A[G])$ and $\psi \in C^{q}(\bar{A}, A[G])$, we have

$$
\mathbf{H}^{*}(\phi \cup \psi)=(-1)^{p} \phi \cup \mathbf{H}^{*}(\psi)+\mathbf{H}^{*}\left(\phi \cup\left(\Upsilon^{*} \circ \Phi^{*}(\psi)\right)\right) .
$$

Proof. Let us introduce the notation $(\cdots]$ for the input such that

$$
\phi\left(a_{0} \otimes \cdots \otimes a_{p}\right]:=\phi\left(a_{0} \otimes \cdots \otimes a_{p-1}\right) a_{p}, \quad \phi \in C^{p}(A, A[G]) .
$$

According to (3.15),

$$
\begin{aligned}
& (-1)^{p} \phi \cup \mathrm{H}^{*}(\psi)\left(a_{1} \otimes \cdots \otimes a_{p} \otimes a_{p+1} \otimes \cdots \otimes a_{p+q-1}\right) \\
= & \sum_{i=1}^{q-1}(-1)^{p+i} \phi\left(a_{1} \otimes \cdots \otimes a_{p}\right) \psi\left(a_{p+1} \otimes \cdots \otimes a_{p+i-1} \otimes \Phi \circ \Upsilon\left(1 \otimes a_{p+i} \otimes \cdots \otimes a_{p+q-1} \otimes 1\right)\right] \\
= & \sum_{i=1}^{q-1}(-1)^{p+i}(\phi \cup \psi)\left(a_{1} \otimes \cdots \otimes a_{p+i-1} \otimes \Phi_{q-i} \circ \Upsilon_{q-i}\left(1 \otimes a_{p+i} \otimes \cdots \otimes a_{p+q-1} \otimes 1\right)\right],
\end{aligned}
$$

and

$$
\begin{aligned}
& \mathrm{H}^{*}\left(\phi \cup\left(\Upsilon^{*} \circ \Phi^{*}(\psi)\right)\right)\left(a_{1} \otimes \cdots \otimes a_{p} \otimes a_{p+1} \otimes \cdots \otimes a_{p+q-1}\right) \\
= & \sum_{i=1}^{p}(-1)^{i}\left(\phi \cup\left(\Upsilon^{*} \circ \Phi^{*}(\psi)\right)\left(a_{1} \otimes \cdots \otimes a_{i-1} \otimes \Phi_{p+q-i} \circ \Upsilon_{p+q-i}\left(1 \otimes a_{i} \otimes \cdots \otimes a_{p+1} \otimes \cdots \otimes a_{p+q-1} \otimes 1\right)\right]\right. \\
& +\sum_{i=1}^{q-1}(-1)^{p+i} \phi\left(a_{1} \otimes \cdots \otimes a_{p}\right) \Upsilon^{*} \circ \Phi^{*}(\psi)\left(a_{p+1} \otimes \cdots \otimes a_{p+i-1} \otimes \Phi_{q-i} \circ \Upsilon_{q-i}\left(1 \otimes a_{p+i} \otimes \cdots \otimes a_{p+q-1} \otimes 1\right)\right] \\
= & \sum_{i=1}^{p}(-1)^{i}(\phi \cup \psi)\left(a_{1} \otimes \cdots \otimes a_{i-1} \otimes \Phi_{p+q-i} \circ \Upsilon_{p+q-i}\left(1 \otimes a_{i} \otimes \cdots \otimes a_{p+1} \otimes \cdots\right)\right) .
\end{aligned}
$$

Here the last equality holds because the second summand vanishes (recall the definition of $\Upsilon$, we pick an increasing splitting for terms in a sequence and put all the latter halves into the first term to make a new sequence, in which we cannot find an new increasing splitting), and $\Upsilon^{*} \circ \Phi^{*}$ will do nothing in the first summand because they will acts on a sequence of terms like $x_{i_{\sigma(1)}} \otimes x_{i_{\sigma(2)}} \cdots$ with $i_{1}<i_{2}<\cdots$.

Sum them up and we will get $\mathrm{H}^{*}(\phi \cup \psi)\left(a_{1} \otimes \cdots \otimes a_{p+q-1}\right)$.

Example 3.1. Consider the case $N=2$ and write $x=x_{1}, y=x_{2}$. For $\phi_{k} \in C^{k}(\bar{A}, A[G])$, we have

$$
\begin{aligned}
& \mathrm{H}_{0}^{*}\left(\phi_{1}\right)=0, \\
& \mathrm{H}_{1}^{*}\left(\phi_{2}\right)\left(x^{a} y^{b}\right)=-\sum_{0 \leqslant s<a} \phi_{2}\left(x^{s} y^{b} \otimes x\right) x^{a-s-1}-\sum_{0 \leqslant t<b} \phi_{2}\left(y^{t} \otimes y\right) x^{a} y^{b-t-1}, \\
& \mathrm{H}_{2}^{*}\left(\phi_{3}\right)\left(x^{a_{1}} y^{b_{1}} \otimes x^{a_{2}} y^{b_{2}}\right) \\
& =-\sum_{\substack{0 \leqslant s<a_{1} \\
0 \leqslant t<b_{2}}} \phi_{3}\left(x^{s} y^{b_{1}+t} \otimes(x \otimes y-y \otimes x)\right) x^{a_{1}+a_{2}-s-1} y^{b_{2}-t-1} \\
& \quad+\sum_{0 \leqslant s<a_{2}} \phi_{3}\left(x^{a_{1}} y^{b_{1}} \otimes x^{s} y^{b_{2}} \otimes x\right) x^{a_{2}-s-1} \\
& \quad+\sum_{0 \leqslant t<b_{2}} \phi_{3}\left(x^{a_{1}} y^{b_{1}} \otimes y^{t} \otimes y\right) x^{a_{2}} y^{b_{2}-t-1} .
\end{aligned}
$$




\subsection{G-twisted cohomology}

Let us now compute the cohomology

$$
\mathrm{HH}_{c}^{\bullet}\left(A_{W}, A_{W}[G]\right)
$$

which is the state space of orbifold Landau-Ginzburg model.

Definition 3.5. Let $g \in G$, $\operatorname{Fix}(g)$ be the fixed locus of $g$. We let

$$
\mathrm{PV}^{\bullet}(\operatorname{Fix}(g))=A_{g} \otimes \wedge^{\bullet} T \operatorname{Fix}(g)
$$

denote algebraic polyvector fields on $\operatorname{Fix}(g)$. Here $T \operatorname{Fix}(g)$ are algebraic vector fields on $\operatorname{Fix}(g)$.

As in the proof of Theorem 2.11 , there is a spectral sequence converging to $\mathrm{HH}_{c}\left(A_{W}, A_{W}[G]\right)$ whose $E_{1}$ page is $\mathrm{HH}_{c}(A, A[G])$. This can be computed by the Koszul resolution

$$
\mathrm{HH}_{c}^{\bullet}(A, A[G]) \simeq \mathrm{H}\left(K^{\bullet}(A, A[G]), \partial_{K}\right) .
$$

Using (3.6), we find (see also [35] and [23])

Lemma 3.6. There is an natural isomorphism between graded vector spaces:

$$
\begin{aligned}
\Theta_{g}: \quad \mathrm{PV}^{\bullet}(\operatorname{Fix}(g))\left[N-N_{g}\right] & \rightarrow \mathrm{HH}_{c}^{\bullet}(A, A g) \simeq \mathrm{H}\left(K^{\bullet}(\bar{A}, A g), \partial_{K}\right) \\
& a \partial_{j_{1}} \wedge \partial_{j_{2}} \wedge \cdots \wedge \partial_{j_{p}} \mapsto a e_{j_{1}} e_{j_{2}} \cdots e_{j_{p}} e_{\boldsymbol{I}_{g}} g .
\end{aligned}
$$

Here $\partial_{i}=\partial / \partial x_{i}$ of degree deg $\partial_{i}=1 . \boldsymbol{I}_{g}=\left\{i_{1}<i_{2}<\cdots<i_{N-N_{g}}\right\}$ is the moving index of $g$ and $e_{\boldsymbol{I}_{g}}:=e_{i_{1}} \cdots e_{i_{N-N g}}$.

Our assumption on $W$ implies that $W_{g}$ has an isolated singularity at the origin (see [15] for details). The complex in the $E_{2}$-page is precisely the Koszul resolution for the critical locus of $W_{g}$. Therefore the spectral sequence converges at $E_{2}$-page whose cohomology in the $g$-sector is $\operatorname{Jac}\left(W_{g}\right)\left[N-N_{g}\right]$.

In conclusion, we have (see also Theorem 4.2. and Theorem 6.3. in [6])

Theorem 3.7. $\Theta_{g}$ in Lemma 3.6 induces a natural isomorphism between $\mathbb{Z} / 2 \mathbb{Z}$-graded vector spaces

$$
\Theta_{g}: \operatorname{Jac}\left(W_{g}\right)\left[\bar{N}-\overline{N_{g}}\right] \cong \mathrm{HH}_{c}^{\bullet}\left(A_{W}, A_{W} g\right) .
$$

Example 3.2 (Fermat type). $A=\mathbb{C}\left[x_{1}\right], W=x_{1}^{n}$. Let $g \in G_{W}$ and $g \neq e$. Then

$$
\mathrm{HH}_{c}\left(A_{W}, A_{W} g\right)=\mathbb{C} e_{1} g
$$

is one-dimensional and has odd parity.

Example 3.3 (Loop type). $A=\mathbb{C}\left[x_{1}, \cdots, x_{N}\right], W=x_{1}{ }^{n_{1}} x_{2}+x_{2}{ }^{n_{2}} x_{3}+\cdots+x_{N}{ }^{n_{N}} x_{1}$. Let $g \in G_{W}$ and $g \neq e$. Assume $g\left(x_{i}\right)=\lambda_{i} x_{i}$. Then $\lambda_{i} \neq 1$ for any $i$. Therefore

$$
\mathrm{HH}_{c}\left(A_{W}, A_{W} g\right)=\mathbb{C} e_{1} e_{2} \cdots e_{N} g
$$

is one-dimensional and has the same parity as $N$.

Example 3.4 (Chain type). $A=\mathbb{C}\left[x_{1}, \cdots x_{N}\right], W=x_{1}^{n_{1}} x_{2}+x_{2}{ }^{n_{2}} x_{3}+\cdots+x_{N}{ }^{n_{N}}$. Let $g \in G_{W}$ and $g \neq e$. Assume $g\left(x_{i}\right)=\lambda_{i} x_{i}$. There exists $l_{g}$ such that $\lambda_{i} \neq 1$ for $i \leq l_{g}$ and $\lambda_{i}=1$ for $i>l_{g}$. Hence

$$
\mathrm{HH}_{c}\left(A_{W}, A_{W} g\right)=\operatorname{Jac}\left(x_{l_{g}+1}^{n_{l_{g}+1}} x_{l_{g}+2}+\cdots+x_{N-1}^{n_{N-1}} x_{N}+x_{N}^{n_{N}}\right) e_{1} \cdots e_{l_{g}} g
$$

whose parity is the same as $l_{g}$. 


\subsection{G-Frobenius algebraic structure}

Definition 3.6 ([29, 30]). A $G$-Frobenius algebra on $\mathbb{C}$ consists of $\left(G, H, \cup, \eta, \rho, 1_{\mathrm{e}}, \chi\right)$, where

1. $G$ is a finite group;

2. $H$ is a finite dimensional $\mathbb{Z}$ (or $\mathbb{Z} / 2 \mathbb{Z}$ )-graded vector space with a sector decomposition

$$
H=\bigoplus_{g \in G} H_{g}
$$

3. $\chi \in \operatorname{Hom}\left(G, \mathbb{C}^{*}\right)$ is a character of $G$,

4. $\rho: G \rightarrow \operatorname{Aut}(H)$ defines a $G$-action on $H$ satisfying

i) $\rho(g): H_{h} \rightarrow H_{g h g^{-1}}$;

ii) $\rho(g): H_{g} \rightarrow H_{g}$ is the scalar multiplication by $\chi(g)^{-1}$;

5. $\eta$ is a non-degenerate bilinear form on $H$ satisfying

i) for $\alpha_{g} \in H_{g}, \alpha_{h} \in H_{h}, \eta\left(\alpha_{g}, \alpha_{h}\right)=0$ unless $g h=\mathrm{e}$,

ii) for $g \in G$ and $\alpha, \beta \in H$,

$$
\eta(\rho(g) \alpha, \rho(g) \beta)=\chi(g)^{-2} \eta(\alpha, \beta) ;
$$

6. $\left(H, \cup, \perp_{\mathrm{e}}\right)$ is an associative algebra with the identity $\perp_{\mathrm{e}}$ satisfying

i) $\cup$ is compatible with the sector decomposition,

$$
\cup: H_{g} \times H_{h} \rightarrow H_{g h},
$$

ii) $\cup$ is $G$-equivariant and $\perp_{\mathrm{e}}$ is $G$-invariant,

iii) $\cup$ is twisted commutative,

$$
\alpha_{g} \cup \rho\left(g^{-1}\right) \alpha_{h}=(-1)^{\left|\alpha_{g}\right|\left|\alpha_{h}\right|} \alpha_{h} \cup \alpha_{g}, \quad \forall \alpha_{g} \in H_{g}, \alpha_{h} \in H_{h},
$$

iv) $\cup$ is compatible with $\eta$,

$$
\eta(\alpha \cup \beta, \gamma)=\eta(\alpha, \beta \cup \gamma), \quad \forall \alpha, \beta, \gamma \in H,
$$

v) the following projective trace axiom holds,

$$
\chi(h) \operatorname{Tr}_{s}\left(\left.\mathrm{~L}_{\alpha} \rho(h)\right|_{H_{g}}\right)=\chi(g)^{-1} \operatorname{Tr}_{s}\left(\left.\rho\left(g^{-1}\right) \mathrm{L}_{\alpha}\right|_{H_{h}}\right), \quad \forall g, h \in G, \alpha \in H_{g h g^{-1} h^{-1}},
$$

where $\mathrm{L}_{\alpha}$ is an operator on $H$ given by

$$
\mathrm{L}_{\alpha}(\beta):=\alpha \cup \beta,
$$

and $\operatorname{Tr}_{s}$ denotes the trace on $\mathbb{Z}$-graded vector spaces and denotes the super trace on $\mathbb{Z} / 2 \mathbb{Z}$ graded vector spaces.

Definition 3.7. A $G$-Frobenius algebra $H$ is called special if

- there exists generator $\mathbf{1}_{g} \in H_{g}$ for any $g \in G$ such that $H_{g}=H_{\mathrm{e}} \cup \mathbf{1}_{g}$, 
- there exists $\rho_{g, h} \in \mathbb{C}^{*}$ for any $g, h \in G$ such that,

$$
\rho(g)\left(\mathbf{1}_{h}\right)=\rho_{g, h} \mathbf{1}_{g h g^{-1}}
$$

In this subsection we show that the state space of orbifold Landau-Ginzburg model

$$
\mathrm{HH}_{c}\left(A_{W}, A_{W}[G]\right)
$$

carries a natural structure of special $G$-Frobenius algebra. This will be constructed in several steps.

Firstly, we have the sector decomposition

$$
\mathrm{HH}_{c}\left(A_{W}, A_{W}[G]\right)=\bigoplus_{g \in G} H_{g}, \quad \text { where } \quad H_{g}=\mathrm{HH}_{c}\left(A_{W}, A_{W} g\right)
$$

together with compatible $G$-action and twisted commutative cup product $\cup$ by Theorem 2.7

Define a character $\chi: G \rightarrow \mathbb{C}^{*}$ of $G$ by

$$
\chi(g)=\operatorname{det}(g)=\lambda_{1}^{g} \lambda_{2}^{g} \cdots \lambda_{N}^{g}, \quad \text { if } \quad{ }^{g} x_{i}=\lambda_{i}^{g} x_{i} .
$$

Lemma 3.8. For any $g \in G$ and $\alpha_{g} \in H_{g}$, we have

$$
g^{*}\left(\alpha_{g}\right)=\chi(g)^{-1} \alpha_{g} .
$$

Proof. This can be checked explicitly using Lemma 3.6 .

Let us now construct the generator $\mathbf{1}_{g} \in H_{g}$ for each $g \in G$. Define

$$
\mathbf{1}_{g}=\Theta_{g}(1) \in H_{g}
$$

where $\Theta_{g}$ is defined in Theorem 3.7 and 1 is the identity in $\operatorname{Jac}\left(W_{g}\right)$. The parity of $\mathbf{1}_{g}$ is the same as $N-N_{g}$. It is easy to see that $\mathrm{\perp}_{\mathrm{e}}$ is the unit of $\mathrm{HH}_{c}\left(A_{W}, A_{W}[G]\right)$. For $g, h \in G$, define

$$
\rho_{g, h}=\prod_{i \in \boldsymbol{I}_{h}}\left(\lambda_{i}^{g}\right)^{-1}
$$

where $\boldsymbol{I}_{h}$ is the moving index of $h$ and $\lambda_{i}^{g}$ is defined by ${ }^{g} x_{i}=\lambda_{i}^{g} x_{i}$. It is checked that

$$
g^{*}\left(\mathbf{1}_{h}\right)=\rho_{g, h} \mathbf{1}_{h}=\rho_{g, h} \mathbf{1}_{g h g^{-1}} .
$$

Let

$$
\Pi_{g}: \operatorname{Jac}(W) \rightarrow \operatorname{Jac}\left(W_{g}\right)
$$

denote the natural restriction map. This is well-defined since $W$ is $g$-invariant.

Lemma 3.9. For any $f \in \operatorname{Jac}(W)$

$$
\left[\Theta_{\mathrm{e}}(f)\right] \cup \mathbf{1}_{g}=\left[\Theta_{g}\left(\Pi_{g}(f)\right)\right] .
$$

In particular, $H_{g}$ is a cyclic $H_{\mathrm{e}}$-module generated by $\mathbf{1}_{g}$. 
Proof. Identity 3.37 follows by checking

$$
\Upsilon^{*}\left(\Theta_{\mathrm{e}}(f)\right) \cup \Upsilon^{*}\left(\Theta_{g}(1)\right)=\Upsilon^{*}\left(\Theta_{g}(f)\right) .
$$

The last statement follows from the surjectivity of $\Pi_{g}$.

We now construct a bilinear form $\eta$ on $\mathrm{HH}_{c}\left(A_{W}, A_{W}[G]\right)$. On the identity sector

$$
H_{\mathrm{e}} \cong \operatorname{Jac}(W)
$$

the pairing $\eta_{\mathrm{e}}$ is defined by the residue

$$
\eta_{\mathrm{e}}\left(\left[\Theta_{\mathrm{e}}\left(f_{1}\right)\right],\left[\Theta_{\mathrm{e}}\left(f_{2}\right)\right]\right)=\operatorname{Res}_{\mathbb{C}^{N}}\left[\begin{array}{c}
f_{1} f_{2} \mathrm{~d} x_{1} \wedge \mathrm{d} x_{2} \wedge \cdots \wedge \mathrm{d} x_{N} \\
\frac{\partial W}{\partial x_{1}} \frac{\partial W}{\partial x_{2}} \cdots \frac{\partial W}{\partial x_{N}}
\end{array}\right] .
$$

Since $W$ has an isolated singularity at the origin, the residue pairing on $\operatorname{Jac}(W)$ is non-degenerate. We extend $\eta_{\mathrm{e}}$ onto twisted sectors by defining

$$
\begin{aligned}
\eta_{g}: H_{g} \otimes H_{g^{-1}} & \rightarrow \mathbb{C}, \\
(\alpha, \beta) & \mapsto \eta_{\mathrm{e}}\left(\alpha \cup \beta, \mathbf{1}_{\mathrm{e}}\right) .
\end{aligned}
$$

Then the bilinear form $\eta$ is defined by

$$
\eta=\sum_{g \in G} \eta_{g}
$$

Lemma 3.10. The bilinear form $\eta$ on $\mathrm{HH}_{c}\left(A_{W}, A_{W}[G]\right)$ is compatible with the cup product

$$
\eta(\alpha \cup \beta, \gamma)=\eta(\alpha, \beta \cup \gamma), \quad \forall \alpha, \beta, \gamma \in H
$$

and satisfies the G-equivariance condition

$$
\eta\left(g^{*}(\alpha), g^{*}(\beta)\right)=\chi(g)^{-2} \eta(\alpha, \beta), \quad \forall \alpha, \beta \in H .
$$

Proof. By construction,

$$
\eta(\alpha \cup \beta, \gamma)=\eta(\alpha, \beta \cup \gamma)=\eta\left(\alpha \cup \beta \cup \gamma, 1_{\mathrm{e}}\right) .
$$

Equation (3.41) follows. Equation (3.42) follows from the $G$-equivariance of $\cup$, the $G$-invariance of $W$ and the property of residue.

The discussion above is summarized as follows.

Theorem 3.11. Let $W$ be a non-degenerate invertible polynomial. Then the state space $\mathrm{HH}_{c}\left(A_{W}, A_{W}[G]\right)$ of orbifold Landau-Ginzburg model together with the $G$-action (2.16), cup product $\cup$, bilinear form $\eta$ (3.40), character $\chi$ (3.31) and generators $\mathbf{1}_{g}$ (3.33) form a special $\mathbb{Z} / 2 \mathbb{Z}$-graded G-Frobenius algebra.

Proof. The proof of (3.28) is via direct calculation by Kaufmann in [29]. Hence, We only need to check the non-degeneracy of $\eta$ on $g$-sectors where $g \neq$ e for elementary invertible polynomials. If $W$ is of Fermat type or loop type, $\mathrm{HH}_{c}\left(A_{W}, A_{W} g\right)$ is one-dimensional (Example 3.2 and Example 3.3. and $\eta\left(\mathbf{1}_{g}, \mathbf{1}_{g^{-1}}\right) \neq 0$ by Theorem 3.20. If $W$ is of chain type, it can be checked directly that $\eta$ on $\mathrm{HH}_{c}\left(A_{W}, A_{W} g\right)$ is proportional to the residue pairing on $\mathrm{Jac}\left(W_{g}\right)$ using Example 3.4, Lemma 3.9 and Theorem 3.20 . 
Remark. We expect that the pairing $\eta$ on $\mathrm{HH}_{c}\left(A_{W}, A_{W} g\right)$ and $\mathrm{HH}_{c}\left(A_{W}, A_{W} g^{-1}\right)$ is the same as the residue pairing in $\operatorname{Jac}\left(W_{g}\right)$ as long as $W_{g}$ has an isolated singularity. Alternatively, there is categorical construction of pairing (Mukai pairing) from the dg-category of matrix factorization [5, 44, 45]. The Mukai pairing of G-equivariant case is explicitly computed in [37]. If one uses the trivial volume form to identity Hochschild homology with cohomology, then our pairing here coincides with their result (see Theorem 4.2.1 in [37] and Theorem 3.20 below). The non-degeneracy of Mukai pairing is proved by Shklyarov in [44] for all homological smooth dg-algebra.

\subsection{Quantum differential operator and cup product}

In this section we establish an explicit formula for cup product of $G$-twisted Hochschild cohomology. This is achieved via the following established quasi-isomorphisms (formula (3.20) and Theorem 3.7)

$$
\left(C^{\bullet}(\bar{A}, A g), \partial_{H}+\mathrm{d}_{W}\right) \underset{\Upsilon^{*}}{\stackrel{\tilde{\Phi}^{*}}{\rightleftarrows}}\left(K^{\bullet}(A, A g), \partial_{K}+\tilde{\mathrm{d}}_{W}\right) \underset{\mathrm{i}}{\stackrel{\mathrm{p}}{\rightleftarrows}}\left(\operatorname{Jac}\left(W_{g}\right)\left[\bar{N}-\overline{N_{g}}\right], 0\right) .
$$

Here $p$ is the natural projection onto the appropriate components of polyvectors.

Given $\alpha_{g} \in \operatorname{Jac}\left(W_{g}\right)\left[\bar{N}-\overline{N_{g}}\right], \beta_{h} \in \operatorname{Jac}\left(W_{h}\right)\left[\bar{N}-\overline{N_{h}}\right]$, their cup product in the $g h$-sector can be computed by

$$
\mathrm{p} \tilde{\Phi}^{*}\left(\left(\Upsilon^{*} \mathrm{i}\left(\alpha_{g}\right)\right) \cup\left(\Upsilon^{*} \mathrm{i}\left(\beta_{h}\right)\right)\right)
$$

For invertible polynomials, it turns out that $U$ on cohomology is determined by the cup product between $g$-sector and $g^{-1}$-sector. In this case $\left(\Upsilon^{*} \mathrm{i}\left(\alpha_{g}\right)\right) \cup\left(\Upsilon^{*} \mathrm{i}\left(\beta_{h}\right)\right)$ is a cocycle in the identity e-sector. It is easy to see that formula (3.43) is reduced to the following

Lemma 3.12. For a $\left(\partial_{H}+\mathrm{d}_{W}\right)$-closed element in the identity e-sector

$$
\phi=\sum_{k=0}^{l} \phi_{2 k}, \quad \phi_{2 k} \in C^{2 k}(\bar{A}, A \mathrm{e}),
$$

we have

$$
\mathrm{p} \circ \tilde{\Phi}^{*}(\phi)=\left[\sum_{k=0}^{l}(-1)^{k}\left(\mathrm{~d}_{W} \mathrm{H}^{*}\right)^{k} \phi_{2 k}\right] .
$$

Here [ ] on the right hand side represents its class in $\mathrm{Jac}(W)$.

We first establish some properties of the cup product $\cup$ and the homotopy operator $\mathrm{H}^{*}$.

Definition 3.8. Given $g=\left(q_{1}, \cdots, q_{N}\right) \in\left(\mathbb{C}^{*}\right)^{N}$, we define the decomposition

$$
g=\prod_{i=1}^{N} g^{(i)}
$$

where $g^{(i)}=\left(1, \cdots, q_{i}, \cdots, 1\right)$ is called the $i$ 'th component of $g$. We define $\tilde{G}$ to be the group generated by $g^{(i)}$ 's for all $g \in G$. 
It would be convenient to extend our Hochschild cochains to $C^{\bullet}(A, A[\tilde{G}])$ and identify $C^{\bullet}(A, A[G])$ as a sub-algebra under the natural embedding

$$
C^{\bullet}(A, A[G]) \hookrightarrow C^{\bullet}(A, A[\tilde{G}]) .
$$

We will also simply write $\phi_{1} \phi_{2}$ for the cup product $\phi_{1} \cup \phi_{2}$ in this subsection.

Recall the quantum differential operator $\partial_{x_{i}}^{g}$ defined by (3.2).

Definition 3.9. For $g \in G$, we define the first order quantum differential operators $\partial_{i}^{g} \in C^{1}\left(A, A g^{(i)}\right)$ by

$$
\partial_{i}^{g}: f \rightarrow \partial_{x_{i}}^{g}(f) g^{(i)}
$$

and the second order quantum differential operators $\partial_{i, j}^{g, h} \in C^{1}(A, A[\tilde{G}])$ by

$$
\partial_{i, j}^{g, h}=\left\{\begin{array}{lll}
\partial_{i}^{g}\left\{\partial_{j}^{h}\right\} & i \neq j & \text { (mixed type) } \\
-\mathrm{H}^{*}\left(\partial_{i}^{g} \partial_{i}^{h}\right) & i=j & \text { (pure type) }
\end{array}\right.
$$

Here $\mathrm{H}^{*}$ is the homotopy on $G$-twisted Hochschild cochains defined in Proposition 3.3 ,

Example 3.5. Let ${ }^{g} x_{i}=\epsilon_{1} x_{i}$ and ${ }^{h} x_{i}=\epsilon_{2} x_{i}$. Recall definition (3.3). We have

$$
\partial_{i, i}^{g, h}\left(x_{i}^{n}\right)=\frac{\epsilon_{1}^{n-1}[n]_{\epsilon_{2}}-[n]_{\epsilon_{1} \epsilon_{2}}}{\epsilon_{1}-1} x_{i}^{n-2} g^{(i)} h^{(i)} .
$$

In parcular, if $\epsilon_{1} \epsilon_{2}=1$, i.e., $g^{(i)}=\left(h^{(i)}\right)^{-1}$,

$$
\partial_{i, i}^{g, h}\left(x_{i}^{n}\right)=\frac{[n]_{\epsilon_{1}}-n}{\epsilon_{1}-1} x_{i}^{n-2} \mathrm{e} .
$$

Using quantum differential operators, the cochain map (3.12)

$$
\Upsilon^{*}: K^{\bullet}(A, A[G]) \rightarrow C^{\bullet}(\bar{A}, A[G])
$$

can be expressed in terms of cup product

$$
\Upsilon^{*}\left(e_{i_{1}} \cdots e_{i_{p}} g\right)=g^{(1)} \cdots g^{\left(i_{1}-1\right)} \partial_{i_{1}}^{g} g^{\left(i_{1}+1\right)} \cdots g^{\left(i_{2}-1\right)} \partial_{i_{2}}^{g} g^{\left(i_{2}+1\right)} \cdots g^{\left(i_{p}-1\right)} \partial_{i_{p}}^{g} g^{\left(i_{p}+1\right)} \cdots g^{(N)},
$$

where $1 \leqslant i_{1}<i_{2}<\cdots<i_{p} \leqslant N$, and $g^{(i)}$ is viewed naturally as an element in $C^{0}\left(\bar{A}, A g^{(i)}\right)$.

Lemma 3.13.

(a) First order quantum differential operators are $\partial_{H}$-closed,

$$
\partial_{H}\left(\partial_{i}^{g}\right)=0 .
$$

(b) Second order quantum differential operators are symmetric

$$
\partial_{i_{1}, i_{2}}^{g, h}=\partial_{i_{2}, i_{1}}^{h, g} .
$$

and satisfy

$$
\partial_{H}\left(\partial_{i, j}^{g, h}\right)= \begin{cases}-\partial_{i}^{g} \partial_{j}^{h}-\partial_{j}^{h} \partial_{i}^{g} & i \neq j \\ -\partial_{i}^{g} \partial_{i}^{h} & i=j .\end{cases}
$$


Proof. (a) is equivalent to the twisted Leibnitz rule

$$
\partial_{i}^{g}(a b)=\partial_{i}^{g}(a) b+a \partial_{i}^{g}(b)=\partial_{x_{i}}^{g}(a)\left(^{g^{(i)}} b\right) g^{(i)}+a \partial_{x_{i}}^{g}(b) g^{(i)}, \quad \forall a, b \in A .
$$

The first equation in (b) is by direct check. The second equation follows from (2.21), (3.50) and (3.17).

The following lemma gives some useful reorganizing rules for the homotopy $\mathrm{H}^{*}$.

Lemma 3.14. Let $\boldsymbol{I}=\left\{i_{1}, i_{2}, \cdots i_{p}\right\}, i_{2}<i_{3}<\cdots<i_{p}$ and $i_{k} \leqslant i_{1}<i_{k+1}$. Then we have the following formula for the homotopy operator

$$
\begin{aligned}
\mathrm{H}^{*}\left(\partial_{i_{1}}^{g_{1}} \partial_{i_{2}}^{g_{2}} \cdots \partial_{i_{p}}^{g_{p}}\right)= & -\partial_{i_{1}, i_{2}}^{g_{1}, g_{2}} \partial_{i_{3}}^{g_{3}} \cdots \partial_{i_{p}}^{g_{p}}-\partial_{i_{2}}^{g_{2}} \partial_{i_{1}, i_{3}}^{g_{1}, g_{3}} \partial_{i_{4}}^{g_{4}} \cdots \partial_{i_{p}}^{g_{p}} \\
& -\cdots-\partial_{i_{2}}^{g_{2}} \cdots \partial_{i_{k-1}}^{g_{k-1}} \partial_{i_{1}, i_{k}}^{g_{1}, g_{k}} \partial_{i_{k+1}}^{g_{k+1}} \cdots \partial_{i_{p}}^{g_{p}} .
\end{aligned}
$$

More generally, if on the left hand side we insert several 0-cochains $h_{1}^{\left(j_{1}\right)}, \cdots, h_{q}^{\left(j_{q}\right)}$ into the product $\partial_{i_{1}}^{g_{1}} \partial_{i_{2}}^{g_{2}} \cdots \partial_{i_{p}}^{g_{p}}$ at positions after $\partial_{i_{1}}^{g_{1}}$, then on the right hand side each term is modified by inserting $h_{1}^{\left(j_{1}\right)}, \cdots, h_{q}^{\left(j_{q}\right)}$ in ascending order as follows: if $i_{k}<j_{s}<i_{k+1}(k \geq 2)$, then $h^{\left(j_{s}\right)}$ is inserted between quantum differential operators indexed by $i_{k}$ and $i_{k+1}$; if $i_{k}=j_{s}<i_{k+1}$, then we combine $h_{s}^{\left(j_{s}\right)}$ and $\partial_{i_{k}}^{g_{k}}$ into $\partial_{i_{k}}^{g_{k} h_{s}^{\left(j_{s}\right)}}$.

Proof. Under the assumption $i_{2}<i_{3}<\cdots<i_{p}$, we have only one term survive in (3.15),

$$
\mathrm{H}^{*}\left(\partial_{i_{1}}^{g_{1}} \partial_{i_{2}}^{g_{2}} \cdots \partial_{i_{p}}^{g_{p}}\right)\left(a_{1} \otimes a_{2} \otimes \cdots \otimes a_{p-1}\right)=-\partial_{i_{1}}^{g_{1}} \partial_{i_{2}}^{g_{2}} \cdots \partial_{i_{p}}^{g_{p}}\left(\Phi \circ \Upsilon\left(1 \otimes a_{1} \otimes \cdots \otimes a_{p-1} \otimes 1\right)\right] .
$$

Here the notation $(\cdots]$ means that

$$
\phi\left(a_{0} \otimes \cdots \otimes a_{p}\right]:=\phi\left(a_{0} \otimes \cdots \otimes a_{p-1}\right) a_{p}, \quad \phi \in C^{p}(A, A[G]) .
$$

Let $\boldsymbol{J}=\left\{i_{2}, \cdots, i_{p-1}\right\}, g=g_{1}^{\left(i_{1}\right)} \cdots g_{p}^{\left(i_{p}\right)}$ and we keep the notations in (3.10). Then

$$
\begin{aligned}
& \mathrm{H}^{*}\left(\partial_{i_{1}}^{g_{1}} \partial_{i_{2}}^{g_{2}} \cdots \partial_{i_{p}}^{g_{p}}\right)\left(a_{1} \otimes a_{2} \otimes \cdots \otimes a_{p-1}\right) \\
= & -\sum_{s \in \mathcal{S}(\boldsymbol{J})} \partial_{i_{1}}^{g_{1}} \partial_{i_{2}}^{g_{2}} \cdots \partial_{i_{p}}^{g_{p}}\left(a_{1}^{1} \cdots a_{p-1}^{1} \otimes x_{i_{2}} \otimes \cdots \otimes x_{i_{p}} \otimes a_{1}^{2} \cdots a_{p-1}^{2}\right] \\
= & -\sum_{\boldsymbol{s} \in \mathcal{S}(\boldsymbol{J})} \partial_{x_{i_{1}}}^{g_{1}}\left(a_{1}^{1} \cdots a_{p-1}^{1}\right)\left({ }^{g}\left(a_{1}^{2} \cdots a_{p-1}^{2}\right)\right) g \\
= & -\sum_{j=1}^{k-1} \partial_{i_{2}}^{g_{2}} \cdots \partial_{i_{j}}^{g_{j}} \partial_{i_{1} i_{j+1}}^{g_{1} g_{j+1}} \partial_{i_{j+2}}^{g_{j+2}} \cdots \partial_{i_{p}}^{g_{p}}\left(a_{1} \otimes a_{2} \otimes \cdots \otimes a_{p-1}\right) .
\end{aligned}
$$

The general case with 0 -cochain insertions is proved similarly.

The next lemma gives certain vanishing conditions for $\mathrm{H}^{*}$ on twisted cochains.

Lemma 3.15. Let $\phi \in C^{\bullet}(A, A[G])$ be a $G$-twisted cochain expressed in terms of cup product of first and second order quantum differential operators and elements of $\tilde{G}$ (viewed as 0 -th cochain). Then $\mathrm{H}^{*} \phi=0$ in the following cases: 
(a) $\phi=\cdots \partial_{i, j}^{g, h}$. has a second order quantum differential operator appearing at the last position;

(b) $\phi$ is of the form of arbitrary insertion of elements of $\tilde{G}$ into the expression

$$
\cdots \partial_{i_{k}, i_{k}^{\prime}}^{g_{k}, g_{k}^{\prime}} \partial_{i_{k+1}}^{g_{k+1}} \cdots \partial_{i_{p-1}}^{g_{p-1}} \partial_{i_{p}}^{g_{p}}
$$

with $i_{k}<i_{k+1}<\cdots<i_{p}$ or $i_{k}^{\prime}<i_{k+1}<\cdots<i_{p}$

(c) $\phi$ is of the form of arbitrary insertion of elements of $\tilde{G}$ into the expression

$$
\partial_{i_{1}}^{g_{1}} \cdots \partial_{i_{p-1}}^{g_{p-1}} \partial_{i_{p}}^{g_{p}}
$$

with $i_{1}<i_{2}<\cdots<i_{p}$

Proof. They are checked directly by (3.10) and (3.15).

We are ready to compute cup product in terms of (3.43) and 3.19). We need to understand how $\tilde{\Phi}^{*}=\Phi^{*}-\Phi^{*}\left(\mathrm{id}+\mathrm{d}_{W} \mathrm{H}^{*}\right)^{-1} \mathrm{~d}_{W} \mathrm{H}^{*}$ acts on cochains of quantum differential operators.

Lemma 3.16. Consider the following cup product of cochains of quantum differential operators

$\phi=a_{1} g^{(1)} \cdots g^{\left(i_{1}-1\right)} \partial_{i_{1}}^{g} g^{\left(i_{1}+1\right)} \cdots \partial_{i_{p}}^{g} g^{\left(i_{p}+1\right)} \cdots g^{(N)} \cup a_{2} h^{(1)} \cdots h^{\left(j_{1}-1\right)} \partial_{j_{1}}^{h} h^{\left(j_{1}+1\right)} \cdots \partial_{j_{q}}^{h} h^{\left(j_{q}+1\right)} \cdots h^{(N)}$

where $i_{1}<i_{2}<\cdots<i_{p}$ and $j_{1}<j_{2}<\cdots<j_{q}$. $a_{1}, a_{2} \in A, g, h \in G$. Then

$$
\begin{aligned}
\mathrm{H}^{*}(\phi)=\sum_{p^{\prime}, j_{k}} & \pm a_{1}^{g} a_{2} g^{(1)} \cdots \partial_{i_{1}}^{g} g^{\left(i_{1}+1\right)} \cdots \partial_{i_{p^{\prime}}}^{g} g^{\left(i_{p^{\prime}}+1\right)} \cdots g^{\left(i_{\left(p^{\prime}+1\right)}-1\right)} \\
& \cup \tilde{h}^{(1)} \cdots \partial_{j_{1}}^{\tilde{h}} \tilde{h}^{\left(j_{1}+1\right)} \cdots \partial_{i_{\left(p^{\prime}+1\right)}^{g}, \tilde{h}}^{\tilde{h}} \tilde{h}^{\left(j_{k}+1\right)} \cdots \partial_{i_{\left(p^{\prime}+2\right)}}^{\tilde{h}} \tilde{h}^{\left(i_{\left(p^{\prime}+2\right)}+1\right)} \cdots \tilde{h}^{(N)} .
\end{aligned}
$$

Here the summation is over all $p^{\prime}<p, j_{k}$ such that $i_{\left(p^{\prime}+1\right)} \geqslant j_{k}$ and $i_{\tilde{p}} \neq j_{l}$ for all $\tilde{p} \geqslant p^{\prime}+2,1 \leqslant l \leqslant q$.

$$
\tilde{h}^{(j)}= \begin{cases}h^{(j)} & \text { if } 1 \leqslant j \leqslant i_{\left(p^{\prime}+1\right)}, \\ h^{(j)} g^{(j)} & \text { if } i_{\left(p^{\prime}+1\right)}<j \leqslant N .\end{cases}
$$

Proof. Assume $j_{l} \leq i_{p}<j_{l+1}$ for some $1 \leq l<q$ or $j_{q} \leq i_{p}$. If not, we consider $i_{p-1}$ and so on. Let

$$
\begin{aligned}
\alpha & =a_{1} g^{(1)} \cdots g^{\left(i_{1}-1\right)} \partial_{i_{1}}^{g} g^{\left(i_{1}+1\right)} \cdots g^{\left(i_{p}-1\right)} \\
\beta & =\partial_{i_{p}}^{g} g^{\left(i_{p}+1\right)} \cdots g^{(N)} \cup a_{2} h^{(1)} \cdots h^{\left(j_{1}-1\right)} \partial_{j_{1}}^{h} h^{\left(j_{1}+1\right)} \cdots \partial_{j_{q}}^{h} h^{\left(j_{q}+1\right)} \cdots h^{(N)} .
\end{aligned}
$$

We apply Lemma 3.5 to $\phi=\alpha \cup \beta$

$$
\mathrm{H}^{*}(\phi)= \pm \alpha \cup \mathrm{H}^{*}(\beta)+\mathrm{H}^{*}\left(\alpha \cup\left(\Upsilon^{*} \circ \Phi^{*}(\beta)\right)\right)
$$

The first term is computed by Lemma 3.14 In the second term, $\Upsilon^{*} \circ \Phi^{*}(\beta)=0$ if $i_{p}=j_{l}$; otherwise the role of $\Upsilon^{*} \circ \Phi^{*}$ amounts to change terms in $\beta$ into the order

$$
\Upsilon^{*} \circ \Phi^{*}(\beta)= \pm \cdots \partial_{j_{1}}^{h} \cdots \partial_{j_{l}}^{h} \cdots \partial_{i_{p}}^{g} \cdots \partial_{j_{l}+1}^{h} \cdots \partial_{j_{q}}^{h} \cdots
$$

Now we apply the same process to $\mathrm{H}^{*}\left(\alpha \cup\left(\Upsilon^{*} \circ \Phi^{*}(\beta)\right)\right)$ but consider $i_{p-1}$. Recursively we arrive at the lemma. 
Theorem 3.17. Consider the cup product between a $g$-sector and $g^{-1}$-sector

$$
\phi=\Upsilon^{*}\left(e_{i_{1}} \cdots e_{i_{p}} g\right) \cup \Upsilon^{*}\left(e_{j_{1}} \cdots e_{j_{q}} g^{-1}\right)
$$

where $i_{1}<i_{2}<\cdots<i_{p}$ and $j_{1}<j_{2}<\cdots<j_{q} . a_{1}, a_{2} \in$ A. Then $\mathrm{p} \circ \tilde{\Phi}^{*}(\phi)$ is computed as follows.

(1) if $p \neq q$, then $\mathrm{p} \circ \tilde{\Phi}^{*}(\phi)=0$.

(2) if $p=q$, consider the following subset of the $p$-th permutation group

$$
V=\left\{\sigma \in S_{p} \mid j_{\sigma(k)} \leq i_{k}, \forall 1 \leq k \leq p\right\} .
$$

Let $g^{(i, j)}=g^{(i)} g^{(i+1)} \cdots g^{(j)}$ for $i \leq j$. Then $\mathrm{p} \circ \tilde{\Phi}^{*}(\phi)$ equals

$$
(-1)^{\frac{p(p-1)}{2}} \sum_{\sigma \in V} \operatorname{sgn}(\sigma) g^{\left(j_{\sigma(1)}, i_{1}-1\right)} \partial_{i_{1}, j_{\sigma(1)}}^{g, g^{-1}}(W)\left(g^{-1}\right)^{\left(j_{\sigma(1)}+1, i_{1}\right)} \cdots g^{\left(j_{\sigma(p)}, i_{p}-1\right)} \partial_{i_{p}, j_{\sigma(p)}}^{g, g^{-1}}(W)\left(g^{-1}\right)^{\left(j_{\sigma(p)}+1, i_{p}\right)}
$$

as elements in $\mathrm{Jac}(W)$.

Proof. We consider the case $p=q$. The case for $p \neq q$ follows by the same steps. Explicitly,

$$
\begin{aligned}
\phi= & a_{1} g^{(1)} \cdots g^{\left(i_{1}-1\right)} \partial_{i_{1}}^{g} g^{\left(i_{1}+1\right)} \cdots \partial_{i_{p}}^{g} g^{\left(i_{p}+1\right)} \cdots g^{(N)} \\
& \cup a_{2}\left(g^{-1}\right)^{(1)} \cdots\left(g^{-1}\right)^{\left(j_{1}-1\right)} \partial_{j_{1}}^{g^{-1}}\left(g^{-1}\right)^{\left(j_{1}+1\right)} \cdots \partial_{j_{p}}^{g^{-1}}\left(g^{-1}\right)^{\left(j_{p}+1\right)} \cdots\left(g^{-1}\right)^{(N)}
\end{aligned}
$$

and by (3.44)

$$
\mathrm{p} \circ \tilde{\Phi}^{*}(\phi)=(-1)^{p}\left(\mathrm{~d}_{W} \mathrm{H}^{*}\right)^{p} \phi .
$$

$\mathrm{H}^{*}(\phi)$ is computed by (3.55). We consider the action of $\mathrm{d}_{W}$ on each term in 3.55). There are two cases:

Case $1 W$ is acted on by some first order quantum differential operators. In this case, there will be a second order quantum differential operator left in the cochain. If we take a further homotopy $\mathrm{H}^{*}$, it will become zero by Lemma $3.15(a),(b)$.

Case $2 W$ is acted on by some second order quantum differential operators. In this case, a further homotopy $\mathrm{H}^{*}$ will bring the cochain back to the form of (3.54) by Lemma 3.14 The relevant second order differential operator is combined by $\partial_{i_{\left(p^{\prime}+1\right)}^{g}}^{g}$ and $\partial_{j_{k}}^{h}$ where $i_{\left(p^{\prime}+1\right)} \geqslant j_{k}$.

Repeating the above steps we arrive at the theorem.

Example 3.6. For $\left.\left.\left.\left.g\right|_{1} \partial_{2}^{g} g\right|_{3} \partial_{4}^{g} \partial_{5}^{g} \cup \partial_{1}^{h} h\right|_{2} \partial_{3}^{h} \partial_{4}^{h} h\right|_{5}$ with $g=h^{-1}$, there are two relevant index maps
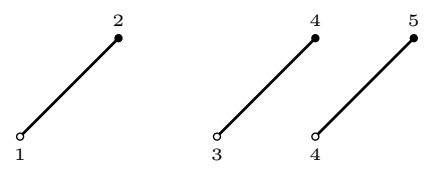

, and
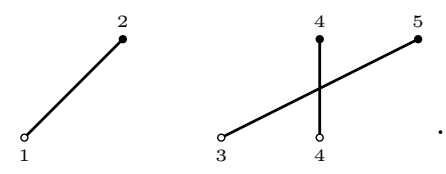

The first one contributes

$$
-g^{(1)} \partial_{2,1}^{g, g^{-1}}(W)\left(g^{-1}\right)^{(2)} g^{(3)} \partial_{4,3}^{g, g^{-1}}(W) \partial_{5,4}^{g, g^{-1}}(W)\left(g^{-1}\right)^{(5)} .
$$

The second one contributes

$$
g^{(1)} \partial_{2,1}^{g, g^{-1}}(W)\left(g^{-1}\right)^{(2)} \partial_{4,4}^{g, g^{-1}}(W) g^{(3)} g^{(4)} \partial_{5,3}^{g, g^{-1}}(W)\left(g^{-1}\right)^{(4)}\left(g^{-1}\right)^{(5)} .
$$




\subsection{Invertible polynomials and cup product formula}

As an application of the method we have developed, we give explicit cup product formula for a general invertible polynomial $W$ and a finite group $G \subseteq G_{W}$. We can write $W=W_{1} \oplus W_{2} \oplus \cdots \oplus W_{r}$ and $G=G_{1} \times G_{2} \times \cdots \times G_{r}$, where $W_{k}$ is an elementary invertible polynomial with dimension $N_{k}$ and $G_{k}$ is a finite subgroup of $G_{W_{k}}$. Also $A=A_{1} \otimes \cdots A_{r}$ is a tensor product of elementary ones.

First of all, we have the following Künneth type formula.

Proposition 3.18. As $\mathbb{Z} / 2 \mathbb{Z}$-graded algebras

$$
\mathrm{HH}_{c}\left(A_{W}, A_{W}[G]\right)=\mathrm{HH}_{c}\left(\left(A_{1}\right)_{W_{1}},\left(A_{1}\right)_{W_{1}}\left[G_{1}\right]\right) \otimes \cdots \otimes \mathrm{HH}_{c}\left(\left(A_{r}\right)_{W_{r}},\left(A_{r}\right)_{W_{r}}\left[G_{r}\right]\right)
$$

where the tensor product on the right hand side is the graded tensor product.

Proof. This is a direct consequence of Theorem 3.7 and the fact that

$$
\operatorname{Jac}(W)=\operatorname{Jac}\left(W_{1}\right) \otimes \cdots \otimes \operatorname{Jac}\left(W_{r}\right) .
$$

This proposition reduces the problem to an elementary invertible polynomial only. Let

$$
\mathbf{1}_{g} \in \mathrm{HH}_{c}\left(A_{W}, A_{W} g\right)
$$

be the generator defined in 3.33).

Lemma 3.19. The cup product on $\mathrm{HH}_{c}^{\bullet}\left(A_{W}, A_{W}[G]\right)$ is completely determined by the generators

$$
\mathbf{1}_{g} \cup \mathbf{1}_{h}, \quad g, h \in G .
$$

Proof. This follows from Lemma 3.9 .

Definition 3.10. Let $W\left(x_{i}\right)$ be an elementary invertible polynomial. Let $g \neq \mathrm{e} \in G_{W}, g\left(x_{i}\right)=\lambda_{i} x_{i}$ and $l_{g}=\sharp\left\{\lambda_{i} \mid \lambda_{i} \neq 1\right\}$. We define the $g$-twisted Hessian, denoted by $\operatorname{Hess}^{g}(W)$, as follows.

(a) If $W=x_{1}^{n}$ is of Fermat type, then

$$
\operatorname{Hess}^{g}(W)=\frac{n}{1-\lambda_{1}} x_{1}^{n-2} .
$$

(b) If $W=x_{1}^{n_{1}} x_{2}+x_{2}^{n_{2}} x_{3}+\cdots+x_{N}^{n_{N}} x_{1}$ is of loop type, then

$$
\operatorname{Hess}^{g}(W)=\frac{(-1)^{N+1}+n_{1} n_{2} \cdots n_{N}}{\left(1-\lambda_{1}\right)\left(1-\lambda_{2}\right) \cdots\left(1-\lambda_{N}\right)} x_{1}^{n_{1}-1} x_{2}^{n_{2}-1} \cdots x_{N}^{n_{N}-1} .
$$

Note that $l_{g}=N$ for any $g \neq e$ in the loop case.

(c) If $W=x_{1}^{n_{1}} x_{2}+x_{2}^{n_{2}} x_{3}+\cdots+x_{N}^{n_{N}}$ is of chain type, then

$$
\operatorname{Hess}^{g}(W)= \begin{cases}\frac{n_{1} n_{2} \cdots n_{l_{g}}}{\left(1-\lambda_{1}\right) \cdots\left(1-\lambda_{l_{g}}\right)} x_{1}^{n_{1}-2} x_{2}^{n_{2}-1} \cdots x_{l_{g}}^{n_{l_{g}}-1} x_{l_{g}+1} & \text { if } l_{g}<N \\ \frac{n_{1} n_{2} \cdots n_{N}}{\left(1-\lambda_{1}\right) \cdots\left(1-\lambda_{N}\right)} x_{1}^{n_{1}-2} x_{2}^{n_{2}-1} \cdots x_{N}^{n_{N}-1} & \text { if } l_{g}=N\end{cases}
$$

Note that $\lambda_{l_{g}+1}=\cdots=\lambda_{N}=1$ for any $g \neq e$ in the chain case. 
Our main theorem in this section is the following.

Theorem 3.20. Let $A$ be a polynomial ring and $W$ be an elementary invertible polynomial. Let $G \subseteq G_{W}$. Then we have the following explicit cup product formula on $\mathrm{HH}_{c}\left(A_{W}, A_{W}[G]\right)$.

(1) For $g_{1}, g_{2} \in G$ and $g_{1}, g_{2} \neq \mathrm{e}, g_{2} \neq g_{1}^{-1}$,

$$
\mathbf{1}_{g_{1}} \cup \mathbf{1}_{g_{2}}=0 \text {. }
$$

(2) For $g \in G$ and $g \neq \mathrm{e}$,

$$
\mathbf{1}_{g} \cup \mathbf{1}_{g^{-1}}=(-1)^{\frac{l_{g}\left(l_{g}-1\right)}{2}} \operatorname{Hess}^{g}(W) \mathbf{1}_{\mathrm{e}} .
$$

Here $l_{g}$ and $\operatorname{Hess}^{g}(W)$ are defined in Definition 3.10

By Proposition 3.18 and Lemma 3.19 this determines the full cup product for all invertible polynomials.

Proof. (1) By Proposition 2.8, we can assume $G=G_{W}$. Let $g_{1}, g_{2} \in G_{W}$ and $g_{1}, g_{2} \neq$ e, $g_{2} \neq g_{1}^{-1}$.

(a) $W=x_{1}^{n}$ is of Fermat type. By Example 3.2, $\mathbf{1}_{g_{1}}$ and $\mathbf{1}_{g_{2}}$ have both odd parity, therefore $\mathbf{1}_{g_{1}} \cup \mathbf{1}_{g_{2}}$ is an even element in the $g_{1} g_{2}$-sector. Since $g_{1} g_{2} \neq 1$, the $g_{1} g_{2}$-twisted sector is generated by $1_{g_{1} g_{2}}$ which is odd. It follows that

$$
\mathbf{1}_{g_{1}} \cup \mathbf{1}_{g_{2}}=0 \text {. }
$$

(b) $W=x_{1}{ }^{n_{1}} x_{2}+x_{2}{ }^{n_{2}} x_{3}+\cdots+x_{N}^{n_{N}} x_{1}$ is of loop type. Then $l_{g_{1}}=l_{g_{2}}=l_{g_{1} g_{2}}=N$. By the $G_{W}$-equivariance of the cup product, we have

$$
h^{*}\left(\mathbf{1}_{g_{1}} \cup \mathbf{1}_{g_{2}}\right)=h^{*}\left(\mathbf{1}_{g_{1}}\right) \cup h^{*}\left(\mathbf{1}_{g_{2}}\right), \quad \forall g \in G_{W},
$$

i.e.

$$
\chi(h)^{-1}\left(\mathbf{1}_{g_{1}} \cup \mathbf{1}_{g_{2}}\right)=\chi(h)^{-2}\left(\mathbf{1}_{g_{1}} \cup \mathbf{1}_{g_{2}}\right),
$$

where $\chi(h)$ is defined in 3.31 . Since $G_{W} \bigcap \mathrm{SL}(N ; \mathbb{C})$ is a proper subgroup of $G_{W}$ (see Proposition 20 in [3]), we can always find an element $h$ such that $\chi(h) \neq 1$. It follows that $\mathbf{1}_{g_{1}} \cup \mathbf{1}_{g_{2}}=0$.

(c) $W=x_{1}^{n_{1}} x_{2}+x_{2}{ }^{n_{2}} x_{3}+\cdots+x_{N}{ }^{n_{N}}$ is of chain type. $x_{1}$ has nontrivial weight under $g_{1}, g_{2}$ and $g_{1} g_{2}$. Consider

$$
h=\operatorname{diag}\left(e^{\frac{2 \pi \sqrt{-1}}{n_{1}}}, 1,1 \cdots 1\right) \in G_{W} .
$$

The equation $h^{*}\left(\mathbf{1}_{g_{1}} \cup \mathbf{1}_{g_{2}}\right)=h^{*}\left(\mathbf{1}_{g_{1}}\right) \cup h^{*}\left(\mathbf{1}_{g_{2}}\right)$ implies that

$$
\exp \left(-\frac{2 \pi \sqrt{-1}}{n_{1}}\right)\left(\mathbf{1}_{g_{1}} \cup \mathbf{1}_{g_{2}}\right)=\exp \left(-\frac{4 \pi \sqrt{-1}}{n_{1}}\right)\left(\mathbf{1}_{g_{1}} \cup \mathbf{1}_{g_{2}}\right) \text {. }
$$

Since $n_{1}>1$, we conclude $\mathbf{1}_{g_{1}} \cup \mathbf{1}_{g_{2}}=0$.

The rest of this paper is devoted to prove part (2) of this theorem.

Remark. We follow the argument in [3] in the proof of part (1) above. 


\section{Fermat type}

$W=x_{1}^{n}$ and $G=\langle\sigma\rangle \cong \mathbb{Z} / n \mathbb{Z}$, where $n \geqslant 2$ and $\sigma=\exp \left(\frac{2 \pi \sqrt{-1}}{n}\right)$. For $1 \leq k<n$, let us first find the representative of $\mathbf{I}_{\sigma^{k}}$ in Koszul cochains. This amounts to extend $e_{1} \sigma^{k}$ into a cochain annihilated by $\partial_{K}+\mathrm{d}_{W}$. It is easy to see that $\mathrm{d}_{W}\left(e_{1} \sigma^{k}\right)=0$, hence

$$
\mathbf{1}_{\sigma^{k}}=e_{1} \sigma^{k}
$$

already does the job. Since $\Upsilon^{*}\left(e_{1} \sigma^{k}\right)=\partial_{1}^{\sigma^{k}}$, we find

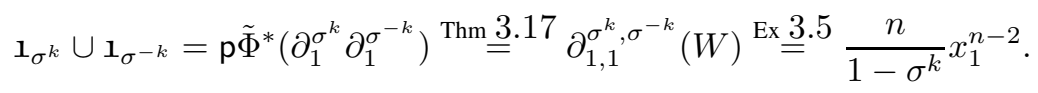

This proves part (2) of Theorem 3.20 in the Fermat case.

\section{Loop type}

$W=x_{1}^{n_{1}} x_{2}+x_{2}^{n_{2}} x_{3}+\cdots+x_{N}^{n_{N}} x_{1}$. Let us first assume $N \geq 3$.

$$
\begin{aligned}
& \text { Let } g=\operatorname{diag}\left(\lambda_{1}, \cdots, \lambda_{N}\right) \in G_{W}, \lambda_{i}=e^{2 \pi \sqrt{-1}} q_{i} \text { where } \\
& \qquad n_{i} q_{i}+q_{i+1} \in \mathbb{Z}, \quad \forall 1 \leq i \leq N, \quad \text { where } q_{N+1} \equiv q_{1} .
\end{aligned}
$$

We require $g \neq e$, then $0<q_{i}<1$ for any $i$. Let us first find the representative of $\mathbf{1}_{g}$ in Koszul cochains. This amounts to extend $\kappa_{0}^{g}=e_{1} e_{2} \cdots e_{N} g$ into $\kappa_{0}^{g}+\kappa_{-2}^{g}+\cdots$ where $\kappa_{-2 k}^{g} \in K^{N-2 k}(A, A g)$ satisfying

$$
\partial_{K}\left(\kappa_{-2 k-2}^{g}\right)+\tilde{\mathrm{d}}_{W}\left(\kappa_{-2 k}^{g}\right)=0 . \quad \text { Then } \mathbf{1}_{g}=\left[\sum_{0 \leq 2 k \leq N} \kappa_{-2 k}^{g}\right] .
$$

For $\boldsymbol{I}=\left\{i_{1}<i_{2}<\cdots i_{m}\right\}$, let us denote $e_{\boldsymbol{I}}:=e_{i_{1}} \cdots e_{i_{k}}$. In the loop case, we have

$$
\tilde{\mathrm{d}}_{W}\left(a e_{\boldsymbol{I}} g\right)=\sum_{k=1}^{p}(-1)^{k-1} a p_{i_{k}}^{g} e_{\boldsymbol{I} \backslash\left\{i_{k}\right\}} g,
$$

where

$$
p_{i}^{g}:= \begin{cases}x_{N}^{n_{N}}+\left[n_{1}\right]_{\lambda_{1}} x_{1}^{n_{1}-1} x_{2} & \text { if } i=1, \\ \lambda_{i-1}^{n_{i-1}} x_{i-1}^{n_{i}-1}+\left[n_{i}\right]_{\lambda_{i}} x_{i}^{n_{i}-1} x_{i+1} & \text { if } 1<i<N, \\ \lambda_{N-1}^{n_{N-1}} x_{N-1}^{n_{N-1}}+\lambda_{1}\left[n_{N}\right]_{\lambda_{N}} x_{N}^{n_{N}-1} x_{1} & \text { if } i=N .\end{cases}
$$

Let us consider the following index set

$$
\mathcal{B}_{s}:=\left\{i_{1}<i_{2}<\cdots<i_{s} \mid i_{k+1}-i_{k}>1, \text { for } 1 \leqslant k<s, \text { and } i_{1}+N-i_{s}>1\right\},
$$

and introduce the notation

$$
b_{i}^{g}:= \begin{cases}\frac{\lambda_{i}^{n_{i}}}{1-\lambda_{i}} x_{i}^{n_{i}-1} & \text { if } 1 \leqslant i<N, \\ \frac{(-1)^{N-1}}{1-\lambda_{N}} x_{N}^{n_{N}-1} & \text { if } i=N .\end{cases}
$$

For each $\boldsymbol{b}=\left\{i_{1}<i_{2}<\cdots<i_{s}\right\} \in \mathcal{B}_{s}$, define

$$
\boldsymbol{I}_{\boldsymbol{b}}:= \begin{cases}\left\{i_{1}, i_{1}+1, i_{2}, i_{2}+1, \cdots i_{s}, i_{s}+1\right\} & \text { while } i_{s} \neq N, \\ \left\{1, i_{1}, i_{1}+1, i_{2}, i_{2}+1, \cdots i_{s}\right\} & \text { while } i_{s}=N .\end{cases}
$$


Lemma 3.21. Let $\boldsymbol{I}_{g}=\{1,2, \cdots, N\}$ and define

$$
\kappa_{-2 s}^{g}:=\sum_{\boldsymbol{b} \in \mathcal{B}_{s}}\left(\prod_{i \in \boldsymbol{b}} b_{i}^{g}\right) e_{\boldsymbol{I}_{g} \backslash \boldsymbol{I}_{\boldsymbol{b}}} g .
$$

Then

$$
\sum_{N \geqslant 2 s \geqslant 0} \kappa_{-2 s}^{g}
$$

gives a Koszul representative for $\mathbf{1}_{g}$.

Proof. For convenience, we will identify index $i$ with $i+N$. For ever $p_{i}^{g}$, we denote

$$
p_{i}^{g}=\left(p_{i}^{g}\right)_{1}+\left(p_{i}^{g}\right)_{2}, \quad \text { where } \quad\left(p_{j}^{g}\right)_{1} \propto x_{j-1}^{n_{j-1}}, \quad\left(p_{j}^{g}\right)_{2} \propto x_{j}^{n_{j}-1} x_{j+1} .
$$

For any index set $\boldsymbol{I} \subset \boldsymbol{I}_{g}$ with $|\boldsymbol{I}|=2 s+1$, denote

$$
\mathcal{H}_{\boldsymbol{I}}=\left\{(\boldsymbol{b}, j) \mid \boldsymbol{b} \in \mathcal{B}_{s}, j \notin \boldsymbol{I}_{\boldsymbol{b}} \text { and } \boldsymbol{I}_{\boldsymbol{b}} \bigcup\{j\}=\boldsymbol{I}\right\} .
$$

For $\boldsymbol{I}$ such that $\mathcal{H}_{\boldsymbol{I}} \neq \emptyset$, let $\boldsymbol{I}_{\mathbf{0}}=\left\{i_{\boldsymbol{I}}, i_{\boldsymbol{I}}+1, \cdots i_{\boldsymbol{I}}+2 \ell_{\boldsymbol{I}}\right\} \subseteq \boldsymbol{I}$, such that $i_{\boldsymbol{I}}-1, i_{\boldsymbol{I}}+2 \ell_{\boldsymbol{I}}+1 \notin \boldsymbol{I}$.Then

$$
\mathcal{H}_{\boldsymbol{I}}=\{(\boldsymbol{b}(\ell), j(\ell))\}_{\ell=0}^{\ell_{\boldsymbol{I}}}, \quad \text { where } \quad j(\ell)=i_{\boldsymbol{I}}+2 \ell .
$$

Note that for $0 \leqslant \ell \leqslant \ell_{\boldsymbol{I}}-1$,

$$
\left(p_{j(\ell)}^{g}\right)_{2} b_{j(\ell)+1}^{g}+b_{j(\ell)}^{g}\left(p_{j(\ell+1)}^{g}\right)_{1}=0 .
$$

Therefore

$$
\begin{aligned}
\tilde{\mathrm{d}}_{W}\left(\kappa_{-2 s}^{g}\right) & =\sum_{\boldsymbol{b} \in \mathcal{B}_{s}} \sum_{j_{k} \notin \boldsymbol{I}_{\boldsymbol{b}}}(-1)^{k-1} p_{j_{k}}^{g}\left(\prod_{i \in \boldsymbol{b}} b_{i}^{g}\right) e_{\boldsymbol{I}_{g} \backslash\left(\boldsymbol{I}_{\boldsymbol{b}} \cup\left\{j_{k}\right\}\right)} g \\
& =\sum_{|\boldsymbol{I}|=2 s+1} \sum_{(\boldsymbol{b}, j) \in \mathcal{H}_{\boldsymbol{I}}}(-1)^{\operatorname{sgn}(\boldsymbol{I})} p_{j}^{g}\left(\prod_{i \in \boldsymbol{b}} b_{i}^{g}\right) e_{\boldsymbol{I}_{g} \backslash \boldsymbol{I}} g \\
& =\sum_{|\boldsymbol{I}|=2 s+1} \sum_{\ell=0}^{\ell_{\boldsymbol{I}}}(-1)^{\operatorname{sgn}(\boldsymbol{I})}\left(\left(p_{j(\ell)}^{g}\right)_{1}+\left(p_{j(\ell)}^{g}\right)_{2}\right)\left(\prod_{i \in \boldsymbol{b}(\ell)} b_{i}^{g}\right) e_{\boldsymbol{I}_{g} \backslash \boldsymbol{I}} g \\
& =\sum_{|\boldsymbol{I}|=2 s+1}(-1)^{\operatorname{sgn}(\boldsymbol{I})}\left(\left(p_{i_{\boldsymbol{I}}}^{g}\right)_{1} \prod_{i \in \boldsymbol{b}(0)} b_{i}^{g}+\left(p_{i_{\boldsymbol{I}}+2 \ell_{\boldsymbol{I}}}^{g}\right)_{2} \prod_{i \in \boldsymbol{b}\left(\ell_{\boldsymbol{I}}\right)} b_{i}^{g}\right) e_{\boldsymbol{I}_{g} \backslash \boldsymbol{I}} g,
\end{aligned}
$$

where $\operatorname{sgn}(\boldsymbol{I})=\sharp\left(\left\{1,2, \cdots i_{\boldsymbol{I}}-1\right\} \backslash \boldsymbol{I}\right)$. Observe

$$
\left(p_{i+1}^{g}\right)_{1}=\left(1-\lambda_{i}\right) x_{i} b_{i}^{g}, \quad-\left(p_{i}^{g}\right)_{2}=\left(1-\lambda_{i+1}\right) x_{i+1} b_{i}^{g},
$$

we have

$$
\begin{aligned}
-\partial_{K}\left(\kappa_{-2 s-2}^{g}\right)= & \sum_{|\boldsymbol{I}|=2 s+1}(-1)^{\operatorname{sgn}(\boldsymbol{I})}\left(\left(1-\lambda_{i_{\boldsymbol{I}}-1}\right) x_{i_{\boldsymbol{I}}-1} \prod_{i \in \boldsymbol{b}(\boldsymbol{I})^{+}} b_{i}^{g}\right. \\
& \left.-\left(1-\lambda_{i_{\boldsymbol{I}}+2 \ell_{\boldsymbol{I}}+1}\right) x_{i_{\boldsymbol{I}}+2 \ell_{\boldsymbol{I}}+1} \prod_{i \in \boldsymbol{b}(\boldsymbol{I})^{-}} b_{i}^{g}\right) e_{\boldsymbol{I}_{g} \backslash \boldsymbol{I}} g
\end{aligned}
$$

where $\boldsymbol{I}_{\boldsymbol{b}(\boldsymbol{I})^{+}}=\boldsymbol{I} \bigcup\left\{i_{\boldsymbol{I}}-1\right\}$ and $\boldsymbol{I}_{\boldsymbol{b}(\boldsymbol{I})^{-}}=\boldsymbol{I} \bigcup\left\{i_{\boldsymbol{I}}+2 \ell_{\boldsymbol{I}}+1\right\}$. We find

$$
\partial_{K}\left(\kappa_{-2 s-2}^{g}\right)+\tilde{\mathrm{d}}_{W}\left(\kappa_{-2 s}^{g}\right)=0 \text {. }
$$


Now we can compute $\mathbf{1}_{g} \cup \mathbf{1}_{g^{-1}}$ by

$$
\mathrm{p} \circ \tilde{\Phi}^{*}\left(\Upsilon^{*}\left(\kappa_{0}^{g}+\kappa_{-2}^{g}+\cdots\right) \cup \Upsilon^{*}\left(\kappa_{0}^{g^{-1}}+\kappa_{-2}^{g^{-1}}+\cdots\right)\right)
$$

The following Lemma is a direct consequence of Theorem 3.17 and Lemma 3.21 .

\section{Lemma 3.22.}

$$
\mathbf{1}_{g} \cup \mathbf{1}_{g^{-1}}=\sum_{\Gamma} \operatorname{Val}(\Gamma)
$$

is a sum of 'values' for all graphs $\Gamma$ with

(a) a set of vertices $V(\Gamma)$ indexed by $1,2, \cdots N$ (coloured by '+') and $1,2, \cdots N$ (coloured by'-');

(b) a set of edges $E(\Gamma)$ of three types such that each vertex has valency 1 :

Type '++': edges having sources coloured + and targets coloured,+ indexed by $+\left(v_{\text {source }}, v_{\text {target }}\right)_{+}$, such that for each edge $+(i, j)_{+}, j \equiv i+1 \bmod N$,

Type '--': edges having sources coloured - and targets coloured - , indexed by _ $\left(v_{\text {source }}, v_{\text {target }}\right)_{-}$, such that for each edge _ $(i, j)_{-}, j \equiv i+1 \bmod N$,

Type '+-': edges having sources coloured + and targets coloured - , indexed by $+\left(v_{\text {source }}, v_{\text {target }}\right)_{-}$, such that for each edge ${ }_{+}(i, j)_{-}, i=j$ or $i=j+1$ or $(i, j)=(N, 1)$,

Given such a graph $\Gamma$, we assign the value of each edge by

$$
\begin{aligned}
& \operatorname{Val}\left({ }_{+}(i, i)_{-}\right)=\frac{\left[n_{i}\right]_{\lambda_{i}}-n_{i}}{\lambda_{i}-1} x_{i}^{n_{i}-2} x_{i+1}, \\
& \operatorname{Val}\left(+(i, i-1)_{-}\right)=\left[n_{i-1}\right]_{\lambda_{i-1}} x_{i-1}^{n_{i-1}-1}, \\
& \operatorname{Val}\left(+(N, 1)_{-}\right)=\left[n_{N}\right]_{\lambda_{N}} x_{N}^{n_{N}-1} \text {, } \\
& \operatorname{Val}\left({ }_{+}(i, i+1)_{+}\right)=b_{i}^{g}= \begin{cases}\frac{\lambda_{i}^{n_{i}}}{1-\lambda_{i}} x_{i}^{n_{i}-1} & \text { while } 1 \leqslant i<N, \\
\frac{(-1)^{N-1}}{1-\lambda_{N}} x_{N}^{n_{N}-1} & \text { while } i=N,\end{cases} \\
& \operatorname{Val}\left({ }_{-}(i, i+1)_{-}\right)={ }^{g} b_{i}^{g^{-1}}= \begin{cases}-\frac{1}{1-\lambda_{i}} x_{i}^{n_{i}-1} & \text { while } 1 \leqslant i<N, \\
\frac{(-1)^{N} \lambda_{N}^{n_{N}}}{1-\lambda_{N}} x_{N}^{n_{N}-1} & \text { while } i=N,\end{cases}
\end{aligned}
$$

and the value of a graph $\Gamma$ is defined by

$$
\operatorname{Val}(\Gamma):=(-1)^{\operatorname{sgn}(\Gamma)+\frac{\left(s_{\Gamma}-1\right) s_{\Gamma}}{2}} \prod_{e \in E(\Gamma)} \operatorname{Val}(e)
$$

Here $s_{\Gamma}$ is the number of edges of type ' +- ' of $\Gamma$. If the type ' +- ' edges have source index $i_{1}<i_{2}<$ $\cdots<i_{s_{\Gamma}}$ and target index $j_{1}<j_{2}<\cdots<j_{s_{\Gamma}}$ such that it connects $i_{k}$ to $j_{\sigma(k)}$, then $(-1)^{\operatorname{sgn}(\Gamma)}$ is the sign of the permutation $\sigma$. 
Example 3.7. For $N=3$, we need to consider the following graphs.

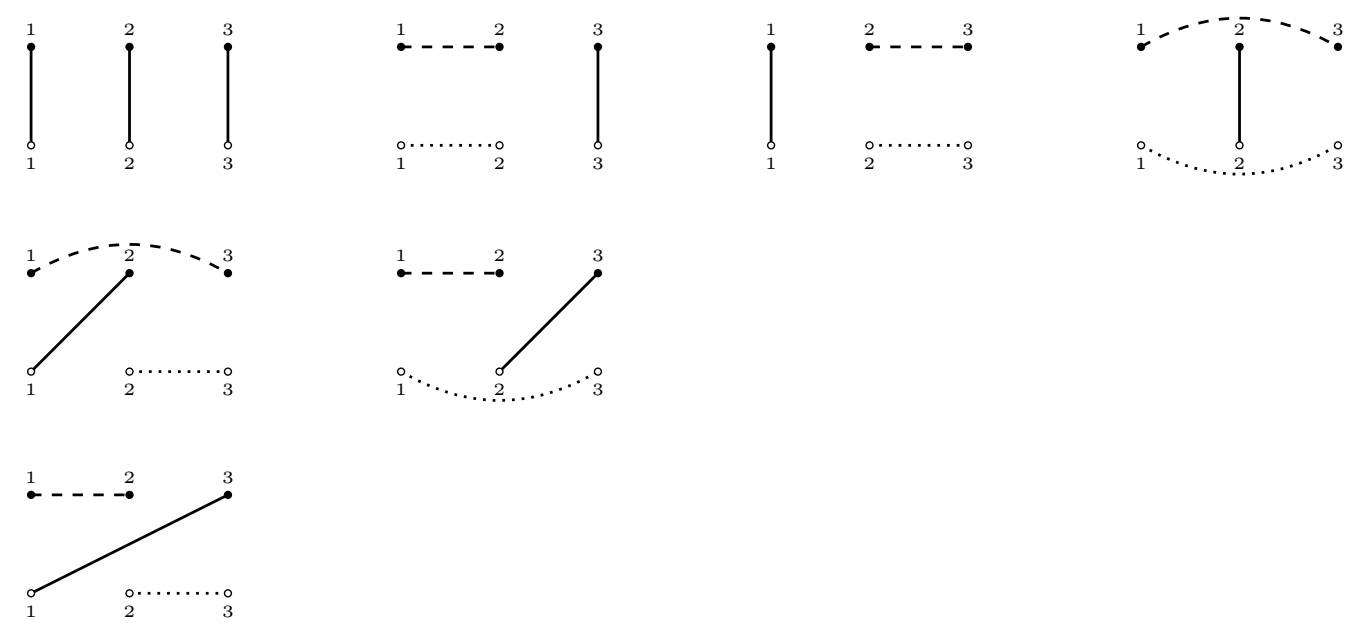

There are three types of graphs in Lemma 3.22

Type I: Graphs with all the type ' +- ' edges indexed by ${ }_{+}(i, i)_{-}$. Then, the other edges comes in pairs;

Type II: Graphs with all the type ' +- ' edges indexed by ${ }_{+}(i, i-1)_{-}$, for $i \neq 1$. Then, the other edges comes in pairs;

Type III: Graphs with all the type ' +- ' edges indexed by ${ }_{+}(i, i-1)_{-}$and by ${ }_{+}(N, 1)_{-}$. Then, there exists edges ${ }_{+}(1,2)_{+}$and ${ }_{-}(N-1, N)_{-}$and the other edges comes in pairs.

We can simplify the graphs by gluing vertices with the same index but different colours, then the three types of graphs look like as follows,
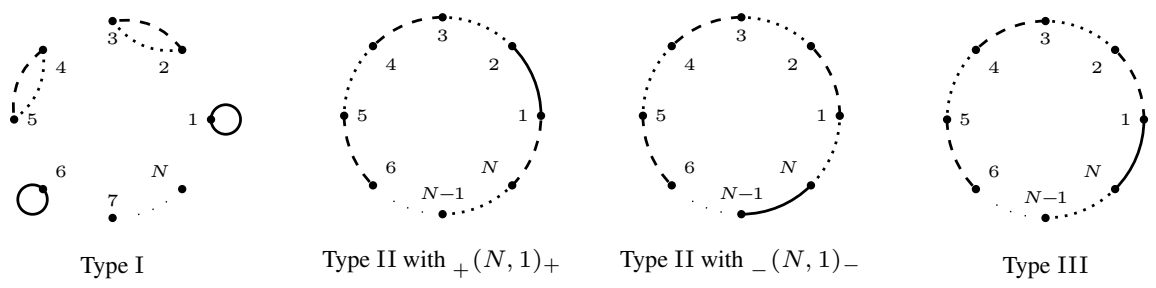

Note that for $1 \leqslant i<N$, we have

$$
\operatorname{Val}\left({ }_{+}(i+1, i)_{-}\right)=\frac{1-\lambda_{i}^{n_{i}}}{1-\lambda_{i}} x_{i}^{n_{i}-1}=-\operatorname{Val}\left(+(i, i+1)_{+}\right)-\operatorname{Val}\left({ }_{-}(i, i+1)_{-}\right),
$$

which means we can replace all these edges of type ' +- ' in graphs of type II by edges of type ' ++ ' of type '--' and multiply by $(-1)^{s_{\Gamma}}$. Hence, graphs with

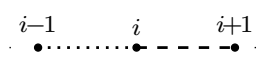


for $2 \leqslant i \leqslant N-1$ will be cancelled and we are left with the graphs

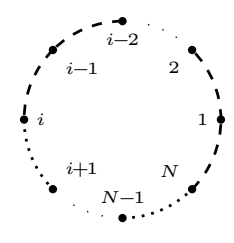

for $1 \leqslant i \leqslant N-1$,

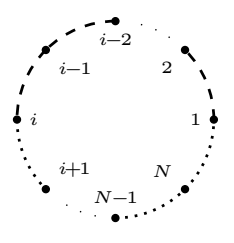

for $2 \leqslant i \leqslant N$.

multiplied by $(-1)^{\frac{(N-1)(N-2)}{2}}$ (for these $\Gamma, \operatorname{sgn}(\Gamma)=0$ and $s_{\Gamma}=N-2$ ).

We can do the same thing to graphs of type III. Then the rest graphs are

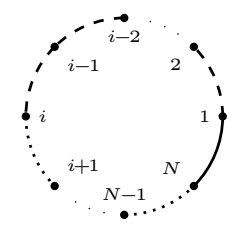

for $2 \leqslant i \leqslant N-1$ and multiplied by $(-1)^{\frac{(N-2)(N-3)}{2}}$ (for these $\Gamma, \operatorname{sgn}(\Gamma)=N-3$ and $s_{\Gamma}=N-2$ ). Since

$$
(-1)^{N} \operatorname{Val}\left({ }_{+}(N, 1)_{-}\right)=-\operatorname{Val}\left({ }_{+}(N, 1)_{+}\right)-\operatorname{Val}\left(\left(_{-}(N, 1)_{-}\right),\right.
$$

the sum of graphs of type II and type III gives only two graphs
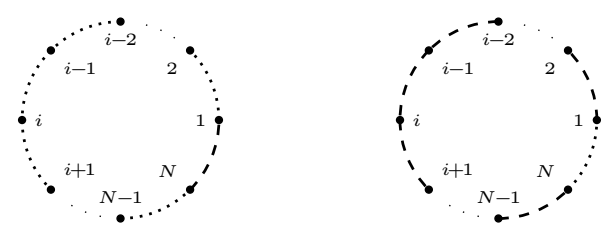

multiplied by $(-1)^{\frac{(N-1)(N-2)}{2}}=(-1)^{\frac{(N-1) N}{2}}(-1)^{N-1}$. Let us consider the matrix

$$
\mathrm{H}_{W}^{g}=\left(\begin{array}{cccccc}
h_{11} & h_{12} & & & & h_{1 N} \\
h_{21} & h_{22} & h_{23} & & & \\
& h_{32} & h_{33} & h_{34} & & \\
& & & \ddots & & \\
& & & & \ddots & h_{(N-1) N} \\
h_{N 1} & & & & h_{N(N-1)} & h_{N N}
\end{array}\right),
$$

where the entries are defined by

$$
h_{i j}:= \begin{cases}\left.\operatorname{Val}_{(+}(i, i)_{-}\right) & \text {if } i=j, \\ \left.\operatorname{Val}_{+}(i, i+1)_{+}\right) & \text {if } N \geqslant j=i+1 \geqslant 2, \\ \left.\operatorname{Val}_{(-}(N, 1)_{-}\right) & \text {if }(i, j)=(N, 1), \\ \left.\operatorname{Val}_{(-}(i-1, i)_{-}\right) & \text {if } N-1 \geqslant j=i-1 \geqslant 1, \\ \left.\operatorname{Val}_{+}(N, 1)_{+}\right) & \text {if }(i, j)=(1, N), \\ 0 & \text { otherwise }\end{cases}
$$


According to the discussion above, the sum of Type I, II, III graphs leads to

$$
\mathbf{1}_{g} \cup \mathbf{1}_{g^{-1}}=(-1)^{\frac{(N-1) N}{2}} \operatorname{det} \mathrm{H}_{W}^{g} .
$$

Note that det $\mathrm{H}_{W}^{g}$ is valued in $\operatorname{Jac}(W)$ where

$$
x_{i-1}^{n_{i-1}}+n_{i} x_{i}^{n_{i}-1} x_{i+1}=0, \quad 1 \leq i \leq N .
$$

Here we always identify indices $N=0, N+1=1$.

The two graphs of type II and type III contribute the following two terms in det $\mathrm{H}_{W}^{g}$

$$
\begin{aligned}
& (-1)^{\frac{(N-1) N}{2}}(-1)^{N-1}\left(h_{12} h_{23} \cdots h_{N 1}+h_{21} h_{32} \cdots h_{1 N}\right) \\
= & (-1)^{\frac{(N-1) N}{2}} \frac{-\left[n_{1}\right]_{\lambda_{1}}\left[n_{2}\right]_{\lambda_{2}} \cdots\left[n_{N}\right]_{\lambda_{N}}-1}{\left(\lambda_{1}-1\right)\left(\lambda_{2}-1\right) \cdots\left(\lambda_{N}-1\right)} x_{1}^{n_{1}-1} \cdots x_{N}^{n_{N}-1} .
\end{aligned}
$$

To compute the contribution of type I graphs, we observe that $h_{i(i+1)}$ and $h_{(i+1) i}$ always come in pairs. The contribution of type I graphs does not change if we replace $h_{i(i+1)}, h_{(i+1) i}$ by other values $\tilde{h}_{i(i+1)}, \tilde{h}_{(i+1) i}$ as long as $h_{i(i+1)} h_{(i+1) i}=\tilde{h}_{i(i+1)} \tilde{h}_{(i+1) i}$ holds for all $1 \leq i \leq N$. In Jac $(W)$, we have

$$
\begin{aligned}
h_{i(i+1)} h_{(i+1) i} & =-\frac{\lambda_{i}^{n_{i}}}{\left(1-\lambda_{i}\right)^{2}} x_{i}^{2 n_{i}-2} \\
& =-\frac{\left(1-\lambda_{i}^{n_{i}}\right) n_{i+1}}{\left(1-\lambda_{i}\right)^{2}\left(1-\lambda_{i+1}\right)} x_{i}^{n_{i}-2} x_{i+1}^{n_{i+1}-1} x_{i+2} \\
& =\left(\frac{\left[n_{i}\right]_{\lambda_{i}}}{\lambda_{i}-1} x_{i}^{n_{i}-2} x_{i+1}\right)\left(-\frac{n_{i+1}}{\lambda_{i+1}-1} x_{i+1}^{n_{i+1}-2} x_{i+2}\right) .
\end{aligned}
$$

Let us replace $h_{i(i+1)}, h_{(i+1) i}$ by

$$
\left\{\begin{array}{l}
\tilde{h}_{i(i+1)}=\frac{\left[n_{i}\right]_{\lambda_{i}}}{\lambda_{i}-1} x_{i}^{n_{i}-2} x_{i+1} \\
\tilde{h}_{(i+1) i}=-\frac{n_{i+1}}{\lambda_{i+1}-1} x_{i+1}^{n_{i+1}-2} x_{i+2}
\end{array}\right.
$$

Observe that

$$
h_{i i}=\tilde{h}_{i(i+1)}+\tilde{h}_{i(i-1)} .
$$

It leads to cancellation of sum of type I graphs that we are left with only two terms

$$
\begin{aligned}
& (-1)^{\frac{(N-1) N}{2}}\left(\tilde{h}_{12} \tilde{h}_{23} \cdots \tilde{h}_{N 1}+\tilde{h}_{21} \tilde{h}_{32} \cdots \tilde{h}_{1 N}\right) \\
= & (-1)^{\frac{(N-1) N}{2}} \frac{\left[n_{1}\right]_{\lambda_{1}}\left[n_{2}\right]_{\lambda_{2}} \cdots\left[n_{N}\right]_{\lambda_{N}}+(-1)^{N} n_{1} n_{2} \cdots n_{N}}{\left(\lambda_{1}-1\right)\left(\lambda_{2}-1\right) \cdots\left(\lambda_{N}-1\right)} x_{1}^{n_{1}-1} \cdots x_{N}^{n_{N}-1} .
\end{aligned}
$$

The sum of all the above contributions proves part (2) of Theorem 3.20 in the loop case for $N \geq 3$. Remark. In $N=2$ case, we can choose

$$
\kappa_{-2}^{g}:=b_{1}^{g}+b_{2}^{g}=\frac{\lambda_{1}^{n_{1}}}{1-\lambda_{1}} x_{1}^{n_{1}-1} g-\frac{1}{1-\lambda_{2}} x_{2}^{n_{2}-1} g,
$$

and define matrix

$$
\mathrm{H}_{W}^{g}=\left(\begin{array}{cc}
\frac{\left[n_{1}\right]_{\lambda_{1}}-n_{1}}{\lambda_{1}-1} x_{1}^{n_{1}-2} x_{2} & \frac{\lambda_{1}^{n_{1}}}{1-\lambda_{1}} x_{1}^{n_{1}-1}-\frac{1}{1-\lambda_{2}} x_{2}^{n_{2}-1} \\
-\frac{1}{1-\lambda_{1}} x_{1}^{n_{1}-1}+\frac{\lambda_{2}^{n_{2}}}{1-\lambda_{2}} x_{2}^{n_{2}-1} & \frac{\left[n_{2}\right]_{\lambda_{2}}-n_{2}}{\lambda_{2}-1} x_{1} x_{2}^{n_{2}-2}
\end{array}\right)
$$


We leave it to the reader to check directly that

$$
\mathbf{I}_{g} \cup \mathbf{I}_{g^{-1}}=\frac{1-n_{1} n_{2}}{\left(\lambda_{1}-1\right)\left(\lambda_{2}-1\right)} x_{1}^{n_{1}-1} x_{2}^{n_{2}-1} .
$$

This proves part (2) of Theorem 3.20 in the loop case for $N=2$.

\section{Chain type}

$W=x_{1}^{n_{1}} x_{2}+x_{2}^{n_{2}} x_{3}+\cdots+x_{N}^{n_{N}}(N \geq 2)$. Let $g=\operatorname{diag}\left(\lambda_{1}, \cdots, \lambda_{N}\right) \in G_{W}, \lambda_{i}=e^{2 \pi \sqrt{-1}} q_{i}$ where

$$
\begin{cases}\lambda_{i}^{n_{i}} \lambda_{i+1}=1 & \text { if } 1 \leqslant i<l_{g} \\ \lambda_{i}^{n_{i}}=1 & \text { if } i=l_{g}, \\ \lambda_{i}=1 & \text { if } l_{g}<i \leqslant N\end{cases}
$$

Here $\lambda_{i} \neq 1$ for $i \leq l_{g}$. Let us find a Koszul representative of $\mathbf{1}_{g}$.

Let $\boldsymbol{I}_{g}=\left\{1,2, \cdots l_{g}\right\}$. For $\boldsymbol{I}=\left\{i_{1}<i_{2}<\cdots i_{m}\right\}$, let us denote $e_{\boldsymbol{I}}:=e_{i_{1}} \cdots e_{i_{k}}$. In the chain case,

$$
\tilde{\mathrm{d}}_{W}\left(\operatorname{age}_{\boldsymbol{I}}\right)=\sum_{k=1}^{p}(-1)^{k-1} a p_{i_{k}}^{g} g e_{\boldsymbol{I} \backslash\left\{i_{k}\right\}},
$$

where

$$
p_{i}^{g}= \begin{cases}{\left[n_{1}\right]_{\lambda_{1}} x_{1}^{n_{1}-1} x_{2}} & \text { if } i=1, \\ \lambda_{i-1}^{n_{i-1}} x_{i-1}^{n_{i-1}}+\left[n_{i}\right]_{\lambda_{i}} x_{i}^{n_{i}-1} x_{i+1} & \text { if } 1<i<l_{g}, \\ \lambda_{l_{g}-1}^{n_{l_{g}-1}} x_{l_{g}-1}^{n_{l_{g}-1}} & \text { if } i=l_{g} .\end{cases}
$$

Let us define the index set

$$
\mathcal{B}_{s}:=\left\{\left\{i_{1}<i_{2}<\cdots<i_{s}\right\} \subset \boldsymbol{I}_{g} \mid i_{k+1}-i_{k}>1 \text {, for } 1 \leqslant k<s, \text { and } i_{s}<l_{g}\right\},
$$

and

$$
b_{i}^{g}=\frac{\lambda_{i}^{n_{i}}}{1-\lambda_{i}} x_{i}^{n_{i}-1}, \quad 1 \leqslant i \leqslant l_{g}-1 .
$$

For each $\boldsymbol{b}=\left\{i_{1}<i_{2}<\cdots<i_{s}\right\} \in \mathcal{B}_{s}$, let

$$
\boldsymbol{I}_{\boldsymbol{b}}=\left\{i_{1}, i_{1}+1, i_{2}, i_{2}+1, \cdots i_{s}, i_{s}+1\right\} .
$$

Then

$$
\sum_{N \geqslant 2 s \geqslant 0} \kappa_{-2 s}^{g}
$$

gives a Koszul representative for $\mathbf{l}_{g}$ where

$$
\kappa_{-2 s}^{g}=\sum_{\boldsymbol{b} \in \mathcal{B}_{s}}\left(\prod_{i \in \boldsymbol{b}} b_{i}^{g}\right) g e_{\boldsymbol{I}_{g} \backslash \boldsymbol{I}_{\boldsymbol{b}}} .
$$

The proof is the same as Lemma 3.21 As in the loop case,

$$
\mathbf{1}_{g} \cup \mathbf{1}_{g^{-1}}=\mathrm{p} \circ \tilde{\Phi}^{*}\left(\Upsilon^{*}\left(\kappa_{0}^{g}+\kappa_{-2}^{g}+\cdots\right) \cup \Upsilon^{*}\left(\kappa_{0}^{g^{-1}}+\kappa_{-2}^{g^{-1}}+\cdots\right)\right) .
$$

is a sum over all the graphs $\Gamma$ with 
(a) a set of vertices $V(\Gamma)$ indexed by $1,2, \cdots l_{g}$;

(b) a set of edges $E(\Gamma)$ of three types such that each vertex has valency 2 :

Type ' ++': edges having sources coloured + and targets coloured + , indexed by $+\left(v_{\text {source }}, v_{\text {target }}\right)_{+}$, satisfying that for each edge ${ }_{+}(i, j)_{+}, j=i+1$,

Type ‘--': edges having sources coloured - and targets coloured - , indexed by _ $\left(v_{\text {source }}, v_{\text {target }}\right)_{-}$, satisfying that for each edge ${ }_{-}(i, j)_{-}, j=i+1$,

Type '+-': edges having sources coloured + and targets coloured - , indexed by $+\left(v_{\text {source }}, v_{\text {target }}\right)_{-}$, satisfying that for each edge ${ }_{+}(i, j)_{-}, i=j$ or $i=j+1$,

Given such a graph $\Gamma$, we assign the value of each edge by

$$
\begin{aligned}
\operatorname{Val}\left(+(i, i)_{-}\right) & = \begin{cases}\frac{\left[n_{i}\right]_{\lambda_{i}}-n_{i}}{\lambda_{i}-1} x_{i}^{n_{i}-2} x_{i+1} & \text { if } 1 \leqslant i<l_{g}, \\
\frac{-n_{l_{g}}}{\lambda_{l_{g}}-1} x_{l_{g}}^{n_{l_{g}}-2} x_{l_{g}+1} & \text { if } i=l_{g}<N, \\
\frac{-n_{l_{g}}}{\lambda_{l_{g}}-1} x_{l_{g}}^{n_{l_{g}}-2} & \text { if } i=l_{g}=N,\end{cases} \\
\operatorname{Val}\left(_{+}(i, i-1)_{-}\right) & =\left[n_{i-1}\right]_{\lambda_{i-1}} x_{i-1}^{n_{i_{-1}-1}},
\end{aligned}
$$

and the value of a graph $\Gamma$ is defined as

$$
\operatorname{Val}(\Gamma)=(-1)^{\operatorname{sgn}(\Gamma)+\frac{\left(s_{\Gamma}-1\right) s_{\Gamma}}{2}} \prod_{e \in E(\Gamma)} \operatorname{Val}(e) .
$$

Here $s_{\Gamma}$ is the number of edges of type ' +- ' of $\Gamma$. If the type ' +- ' edges have source index $i_{1}<i_{2}<$ $\cdots<i_{s_{\Gamma}}$ and target index $j_{1}<j_{2}<\cdots<j_{s_{\Gamma}}$ such that it connects $i_{k}$ to $j_{\sigma(k)}$, then $(-1)^{\operatorname{sgn}(\Gamma)}$ is the sign of the permutation $\sigma$.

Example 3.8. For $l_{g}=3$, we need to consider the following graphs.

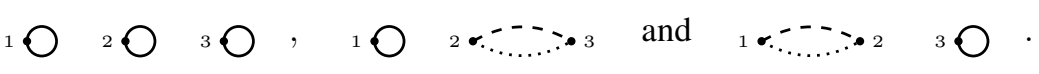

Graphs for chain types are those of type I graphs for the loop case.

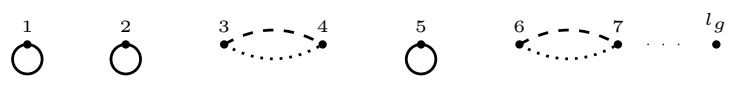

Similar to the discussion for the loop case, let us define the matrix

$$
\mathrm{H}_{W}^{g}=\left(\begin{array}{cccccc}
h_{11} & h_{12} & & & & \\
h_{21} & h_{22} & h_{23} & & & \\
& h_{32} & h_{33} & h_{34} & & \\
& & & \ddots & & \\
& & & & \ddots & h_{\left(l_{g}-1\right) l_{g}} \\
& & & & h_{l_{g}\left(l_{g}-1\right)} & h_{l_{g} l_{g}}
\end{array}\right)
$$


where the entries are defined by

$$
h_{i j}= \begin{cases}\operatorname{Val}\left({ }_{+}(i, i)_{-}\right) & \text {if } i=j, \\ \operatorname{Val}\left(+(i, i+1)_{+}\right) & \text {if } l_{g} \geqslant j=i+1 \geqslant 2, \\ \operatorname{Val}\left({ }_{-}(i-1, i)_{-}\right) & \text {if } l_{g}-1 \geqslant j=i-1 \geqslant 1, \\ 0 & \text { otherwise. }\end{cases}
$$

Then we have the analogue of 3.66 in the chain case

$$
\mathbf{1}_{g} \cup \mathbf{1}_{g^{-1}}=(-1)^{\frac{\left(l_{g}-1\right) l_{g}}{2}} \operatorname{det} \mathrm{H}_{W}^{g} .
$$

The computation of this determinant is also the same as in the loop case for type I graphs. In Jac $(W)$,

$$
\left\{\begin{array}{l}
x_{i-1}^{n_{i-1}}+n_{i} x_{i}^{n_{i}-1} x_{i+1}=0 \quad \text { for } 2 \leqslant i \leqslant N-1 \\
x_{N-1}^{n_{N-1}}+n_{N} x_{N}^{n_{N}-1}=0 .
\end{array}\right.
$$

For $l_{g}<N$, since ${ }_{+}(i, i+1)_{+}$and ${ }_{-}(i, i+1)_{-}$come in pairs and

$$
\begin{aligned}
h_{i(i+1)} h_{(i+1) i} & =-\frac{\lambda_{i}^{n_{i}}}{\left(1-\lambda_{i}\right)^{2}} x_{i}^{2 n_{i}-2} \\
& =-\frac{\left(1-\lambda_{i}^{n_{i}}\right) n_{i+1}}{\left(1-\lambda_{i}\right)^{2}\left(1-\lambda_{i+1}\right)} x_{i}^{n_{i}-2} x_{i+1}^{n_{i+1}-1} x_{i+2} \\
& =\left(\frac{\left[n_{i}\right]_{\lambda_{i}}}{\lambda_{i}-1} x_{i}^{n_{i}-2} x_{i+1}\right)\left(-\frac{n_{i+1}}{\lambda_{i+1}-1} x_{i+1}^{n_{i+1}-2} x_{i+2}\right) .
\end{aligned}
$$

Without changing of the value of det $\mathrm{H}_{W}^{g}$, we can replace $h_{i(i+1)}$ and $h_{(i+1) i}$ by

$$
\begin{aligned}
\tilde{h}_{i(i+1)} & =\frac{\left[n_{i}\right]_{\lambda_{i}}}{\lambda_{i}-1} x_{i}^{n_{i}-2} x_{i+1}, \\
\tilde{h}_{(i+1) i} & =-\frac{n_{i+1}}{\lambda_{i+1}-1} x_{i+1}^{n_{i+1}-2} x_{i+2} .
\end{aligned}
$$

Observe that

$$
h_{i i}= \begin{cases}\tilde{h}_{i(i+1)}+\tilde{h}_{(i-1) i} & \text { while } 2 \leqslant i \leqslant l_{g}-1, \\ \tilde{h}_{l_{g}\left(l_{g}-1\right)} & \text { while } i=l_{g} .\end{cases}
$$

It leads to cancellation of sum of graphs that we are left with only two terms

$$
\begin{aligned}
& (-1)^{\frac{\left(l_{g}-1\right) l_{g}}{2}}\left(\left(h_{11}-\tilde{h}_{12}\right) \tilde{h_{21}} \cdots \tilde{h}_{l_{g}\left(l_{g}-1\right)}\right) \\
= & (-1)^{\frac{\left(l_{g}-1\right) l_{g}}{2}} \frac{n_{1} n_{2} \cdots n_{l_{g}}}{\left(1-\lambda_{1}\right) \cdots\left(1-\lambda_{l_{g}}\right)} x_{1}^{n_{1}-2} x_{2}^{n_{2}-1} \cdots x_{l_{g}}^{n_{l_{g}}-1} x_{l_{g}+1} .
\end{aligned}
$$

For $l_{g}=N$, the computation is similar. This proves part (2) of Theorem 3.20 in the chain case.

\section{References}

[1] Arnol'd, V.I., S.M. Guseı̆n-Zade, and A.N. Varchenko, "Singularities of differentiable maps. Vol. I." Monographs in Mathematics 82 (1985). 
[2] Baranovsky, Vladimir, "Orbifold Cohomology as Periodic Cyclic Homology.” International Journal of Mathematics 14.08 (2003): 791-812.

[3] Basalaev, Alexey, Atsushi Takahashi, and Elisabeth Werner, "Orbifold Jacobian algebras for invertible polynomials." arXiv preprint arXiv:1608.08962 (2016).

[4] Berglund, Per, and Tristan Hübsch, "A Generalized Construction of Mirror Manifolds." Nuclear Physics (1993): 377-391.

[5] Căldăraru, Andrei, and Simon Willerton. "The Mukai pairing. I. A categorical approach.” New York J. Math 16 (2010): 61-98.

[6] Căldăraru, Andrei, and Junwu Tu, "curved $A_{\infty}$-algebras and Landau-Ginzburg models." New York J. Math 19 (2013): 305-342.

[7] Chas, Moira, and Dennis Sullivan, "String topology." arXiv preprint math/9911159 (1999).

[8] Chiodo, Alessandro, and Yongbin Ruan, "LG/CY correspondence: the state space isomorphism." Advances in Mathematics 227.6 (2011): 2157-2188.

[9] Chiodo, Alessandro, Hiroshi Iritani, and Yongbin Ruan, "Landau-Ginzburg/Calabi-Yau correspondence, global mirror symmetry and Orlov equivalence." Publications Mathématiques de l'IHÉS 119.1 (2014): 127-216.

[10] Crainic, Marius, "On the perturbation lemma, and deformations." arXiv preprint math/0403266 (2004).

[11] Dolgushev, Vasiliy, and Pavel Etingof, "Hochschild cohomology of quantized symplectic orbifolds and the Chen-Ruan cohomology.” International Mathematics Research Notices 2005.27 (2005): $1657-1688$.

[12] Dyckerhoff, Tobias, "Compact generators in categories of matrix factorizations." Duke Mathematical Journal 159.2 (2011): 223-274.

[13] Ebeling, Wolfgang, and Atsushi Takahashi, "Variance of the exponents of orbifold Landau-Ginzburg models." Mathematical Research Letters 20.1 (2013): 51-65.

[14] Eisenbud, David, "Homological algebra on a complete intersection, with an application to group representations." Transactions of the American Mathematical Society 260.1 (1980): 35-64.

[15] Fan, Huijun, Tyler Jarvis, and Yongbin Ruan, "The Witten equation, mirror symmetry, and quantum singularity theory." Annals of Mathematics 178.1 (2013): 1-106.

[16] Gerstenhaber, Murray, "The cohomology structure of an associative ring." Annals of Mathematics (1963): 267-288.

[17] Gerstenhaber, Murray, and Alexander A. Voronov, "Homotopy G-algebras and moduli space operad." arXiv preprint hep-th/9409063 (1994).

[18] Getzler, Ezra, and John D. S. Jones, " $A_{\infty}$-algebras and the cyclic bar complex.” Illinois J. Math 34.2 (1990): 256-283.

[19] Getzler, Ezra, "Cartan homotopy formulas and the Gauss-Manin connection in cyclic homology." Israel Math. Conf. Proc. Vol. 7. 1993. 
[20] Getzler, Ezra, and John D. S. Jones, "Operads, homotopy algebra and iterated integrals for double loop spaces.” arXiv preprint hep-th/9403055 (1994).

[21] Givental, Alexander B., "Semisimple Frobenius structures at higher genus." International mathematics research notices 2001.23 (2001): 1265-1286.

[22] Griffiths, Phillip, and Joseph Harris, “Principles of algebraic geometry.” John Wiley \& Sons, 2014.

[23] Halbout, Gilles, and Xiang Tang, "Noncommutative Poisson structures on orbifolds.” Transactions of the American Mathematical Society 362.5 (2010): 2249-2277.

[24] He, Weiqiang, S. Li, Y. Shern, and R. Webb, "Landau-Ginzburg Mirror Symmetry Conjecture." arXiv preprint arXiv:1503.01757(2015).

[25] Husemoller, Dale, John C. Moore, and James Stasheff, "Differential homological algebra and homogeneous spaces.” Journal of Pure and Applied Algebra 5.2 (1974): 113-185.

[26] Intriligator, Kenneth, and Cumrun Vafa, "Landau-Ginzburg orbifolds." Nuclear Physics B 339.1 (1990): 95-120.

[27] Kapustin, Anton, and Y. Li. “D-Branes in Landau-Ginzburg Models and Algebraic Geometry.” Journal of High Energy Physics 312.12(2002):569-588.

[28] Kapustin, Anton, and Y. Li. "Topological Correlators in Landau-Ginzburg Models with Boundaries." Advances in Theoretical \& Mathematical Physics 7.4(2003):727-749.

[29] Kaufmann, Ralph M., “Orbifolding Frobenius algebras.” International Journal of Mathematics 14.06 (2003): 573-617.

[30] Kaufmann, Ralph M., "Singularities with symmetries, orbifold Frobenius algebras and mirror symmetry." Contemporary Mathematics 403 (2006): 67.

[31] Krawitz, Marc, "FJRW rings and Landau-Ginzburg mirror symmetry." arXiv: Algebraic Geometry (2009).

[32] Kreuzer, Maximillian, and Harald Skarke, "On the classification of quasihomogeneous functions." Communications in Mathematical Physics 150.1 (1992): 137-147.

[33] Kreuzer, Maximilian, "The mirror map for invertible LG models.” Physics Letters B, 328.3-4 (1994): 312-318.

[34] Loday, Jean-Louis, “Cyclic homology.” Vol. 301. Springer Science \& Business Media, 2013.

[35] Neumaier, Nikolai, et al., "Homology of formal deformations of proper étale Lie groupoids.” Journal fur die reine und angewandte Mathematik (Crelles Journal) 2006.593 (2006): 117-168.

[36] Polishchuk, Alexander, and Leonid Positselski, "Hochschild (co) homology of the second kind I." Transactions of the American Mathematical Society 364.10 (2012): 5311-5368.

[37] Polishchuk, Alexander, and Arkady Vaintrob, "Chern characters and Hirzebruch-Riemann-Roch formula for matrix factorizations.” Duke Mathematical Journal 161.10 (2012): 1863-1926.

[38] Positselski, Leonid, "Two kinds of derived categories, Koszul duality, and comodule-contramodule correspondence.” American Mathematical Soc., 2011. 
[39] Saito, Kyoji, "Period mapping associated to a primitive form." Publications of the Research Institute for Mathematical Sciences 19.3 (1983): 1231-1264.

[40] Segal, Ed. "The closed state space of affine Landau-Ginzburg B-models.” Journal of Noncommutative Geometry 7.3 (2013): 857-883.

[41] Shepler, Anne V., and Sarah Witherspoon, "Finite groups acting linearly: Hochschild cohomology and the cup product." Advances in Mathematics 226.4 (2011): 2884-2910.

[42] Shepler, Anne V., and Sarah Witherspoon, "Quantum differentiation and chain maps of bimodule complexes.” Algebra \& Number Theory 5.3 (2011): 339-360.

[43] Shepler, Anne V., and Sarah Witherspoon, "Group actions on algebras and the graded Lie structure of Hochschild cohomology.” Journal of Algebra 351.1 (2012): 350-381.

[44] Shklyarov, D., "Hirzebruch Riemann Roch type formula for DG algebras." Proceedings of the London Mathematical Society 106(2013).

[45] Shklyarov, D., "Matrix factorizations and higher residue pairings." Advances in Mathematics (2016): 181-209.

[46] Shklyarov, D., “On Hochschild invariants of Landau-Ginzburg orbifolds." arXiv preprint arXiv:1708.06030(2017).

[47] Stasheff, James Dillon, "Homotopy associativity of H-spaces. II.” Transactions of the American Mathematical Society 108.2 (1963): 293-312.

[48] Stefan, D., "Hochschild cohomology on Hopf Galois extensions." Journal of Pure \& Applied Algebra 103.2(1995):221-233.

[49] Tsygan, Boris, "Cyclic homology." Cyclic Homology in Non-Commutative Geometry. Springer Berlin Heidelberg, 2004. 73-113.

[50] Tu, Junwu, "Matrix factorizations via Koszul duality." Compositio Mathematica 150.09 (2014): 1549-1578.

[51] Vafa, Cumrun, "String vacua and orbifoldized LG models." Modern Physics Letters A 04.12 (1989): 1169-1185.

[52] Voronov, Alexander A., and Murray Gerstenhaber, "Higher operations on the Hochschild complex." Functional Analysis and its Applications 29.1 (1995): 1-5.

[53] Weibel, Charles A., "An introduction to homological algebra." No. 38. Cambridge university press, 1995.

\footnotetext{
Weiqiang He, YMSC, Tsinghua University, BEIJING 100084, China

E-mail address: wqhe@math.tsinghua.edu.cn

Si Li, YMSC, Tsinghua University, Beijing 100084, China

E-mail address: sili@mail.tsinghua.edu.cn

Yifan Li, YMSC, TsinghuA UNIVERSITY, BEIJING 100084, CHINA

E-mail address: yf-li14@mails.tsinghua.edu.cn
} 